Supporting Information for Crystal Growth \& Design

Homochiral Metal-Organic Frameworks of Lead(II) and Cadmium(II) Constructed by Amino Acid-Functionalized Isophthalic Acid: Synthesis, Structure Diversity, and Optical Properties

Yafang Ge,${ }^{\dagger}$ Baoshan Teng, Lulu Lv, ${ }^{\dagger}$ Rui Chen,${ }^{\dagger}$ and Benlai $\mathrm{Wu}^{*, \dagger}$ $\dagger$ College of Chemistry, Zhengzhou University, Zhengzhou 450001, P. R. China

Table S1. Selected Bond Lengths $(\AA)$ and Angles $\left({ }^{\circ}\right)$ for 1-4

\begin{tabular}{|c|c|c|c|}
\hline & & & \\
\hline $\mathrm{Pb} 1-\mathrm{O} 2 \mathrm{~A}$ & $2.494(18)$ & $\mathrm{Pb} 1-\mathrm{O} 3$ & $2.515(9)$ \\
\hline $\mathrm{Pb} 1-\mathrm{O} 6 \mathrm{~B}$ & $2.563(17)$ & $\mathrm{Pb} 1-\mathrm{O} 4$ & $2.577(15)$ \\
\hline $\mathrm{Pb} 1-\mathrm{O} 1 \mathrm{~A}$ & $2.635(16)$ & $\mathrm{Pb} 1-\mathrm{O} 5 \mathrm{~B}$ & $2.719(14)$ \\
\hline $\mathrm{Pb} 2-\mathrm{O} 7 \mathrm{C}$ & $2.516(17)$ & $\mathrm{Pb} 2-\mathrm{O} 10$ & $2.519(15)$ \\
\hline $\mathrm{Pb} 2-\mathrm{O} 11 \mathrm{D}$ & $2.523(15)$ & $\mathrm{Pb} 2-\mathrm{O} 8 \mathrm{C}$ & $2.676(16)$ \\
\hline $\mathrm{Pb} 2-\mathrm{O} 12 \mathrm{D}$ & $2.703(14)$ & $\mathrm{Pb} 2-\mathrm{O} 9$ & $2.704(12)$ \\
\hline $\mathrm{O} 2 \mathrm{~A}-\mathrm{Pb} 1-\mathrm{O} 3$ & $127.7(5)$ & $\mathrm{O} 2 \mathrm{~A}-\mathrm{Pb} 1-\mathrm{O} 6 \mathrm{~B}$ & $80.6(7)$ \\
\hline $\mathrm{O} 3-\mathrm{Pb} 1-\mathrm{O} 6 \mathrm{~B}$ & $84.8(7)$ & $\mathrm{O} 2 \mathrm{~A}-\mathrm{Pb} 1-\mathrm{O} 4$ & $76.8(5)$ \\
\hline $\mathrm{O} 3-\mathrm{Pb} 1-\mathrm{O} 4$ & $51.9(4)$ & $\mathrm{O} 6 \mathrm{~B}-\mathrm{Pb} 1-\mathrm{O} 4$ & $84.1(6)$ \\
\hline $\mathrm{O} 2 \mathrm{~A}-\mathrm{Pb} 1-\mathrm{O} 1 \mathrm{~A}$ & $49.8(5)$ & $\mathrm{O} 3-\mathrm{Pb} 1-\mathrm{O} 1 \mathrm{~A}$ & $161.5(4)$ \\
\hline $\mathrm{O} 6 \mathrm{~B}-\mathrm{Pb} 1-\mathrm{O} 1 \mathrm{~A}$ & $76.7(7)$ & $\mathrm{O} 4-\mathrm{Pb} 1-\mathrm{O} 1 \mathrm{~A}$ & $125.1(4)$ \\
\hline $\mathrm{O} 2 \mathrm{~A}-\mathrm{Pb} 1-\mathrm{O} 5 \mathrm{~B}$ & $117.3(8)$ & $\mathrm{O} 3-\mathrm{Pb} 1-\mathrm{O} 5 \mathrm{~B}$ & $87.1(9)$ \\
\hline $\mathrm{O} 6 \mathrm{~B}-\mathrm{Pb} 1-\mathrm{O} 5 \mathrm{~B}$ & $48.9(5)$ & $\mathrm{O} 4-\mathrm{Pb} 1-\mathrm{O} 5 \mathrm{~B}$ & $122.1(7)$ \\
\hline $\mathrm{O} 1 \mathrm{~A}-\mathrm{Pb} 1-\mathrm{O} 5 \mathrm{~B}$ & $80.5(9)$ & $\mathrm{O} 7 \mathrm{C}-\mathrm{Pb} 2-\mathrm{O} 10$ & $74.1(5)$ \\
\hline $\mathrm{O} 7 \mathrm{C}-\mathrm{Pb} 2-\mathrm{O} 11 \mathrm{D}$ & $80.4(6)$ & $\mathrm{O} 10-\mathrm{Pb} 2-\mathrm{O} 11 \mathrm{D}$ & $85.5(5)$ \\
\hline $\mathrm{O} 7 \mathrm{C}-\mathrm{Pb} 2-\mathrm{O} 8 \mathrm{C}$ & $51.6(5)$ & $\mathrm{O} 10-\mathrm{Pb} 2-\mathrm{O} 8 \mathrm{C}$ & $125.5(4)$ \\
\hline $\mathrm{O} 11 \mathrm{D}-\mathrm{Pb} 2-\mathrm{O} 8 \mathrm{C}$ & $83.1(6)$ & $\mathrm{O} 7 \mathrm{C}-\mathrm{Pb} 2-\mathrm{O} 12 \mathrm{D}$ & $118.9(7)$ \\
\hline
\end{tabular}




\begin{tabular}{|c|c|c|c|}
\hline $\mathrm{O} 10-\mathrm{Pb} 2-\mathrm{O} 12 \mathrm{D}$ & $123.2(6)$ & $\mathrm{O} 11 \mathrm{D}-\mathrm{Pb} 2-\mathrm{O} 12 \mathrm{D}$ & $49.0(4)$ \\
\hline $\mathrm{O} 8 \mathrm{C}-\mathrm{Pb} 2-\mathrm{O} 12 \mathrm{D}$ & $86.0(7)$ & $\mathrm{O} 7 \mathrm{C}-\mathrm{Pb} 2-\mathrm{O} 9$ & $118.4(5)$ \\
\hline $\mathrm{O} 10-\mathrm{Pb} 2-\mathrm{O} 9$ & $49.0(4)$ & $\mathrm{O} 11 \mathrm{D}-\mathrm{Pb} 2-\mathrm{O} 9$ & $74.4(6)$ \\
\hline $\mathrm{O} 8 \mathrm{C}-\mathrm{Pb} 2-\mathrm{O} 9$ & $157.0(4)$ & $\mathrm{O} 12 \mathrm{D}-\mathrm{Pb} 2-\mathrm{O} 9$ & $82.8(7)$ \\
\hline \multicolumn{4}{|c|}{2} \\
\hline $\mathrm{Pb} 1-\mathrm{O} 4 \mathrm{~A}$ & $2.45(2)$ & $\mathrm{Pb} 1-\mathrm{O} 1$ & $2.47(2)$ \\
\hline $\mathrm{Pb} 1-\mathrm{O} 3 \mathrm{~A}$ & $2.51(2)$ & $\mathrm{Pb} 1-\mathrm{O} 6 \mathrm{~B}$ & $2.54(2)$ \\
\hline $\mathrm{Pb} 1-\mathrm{O} 2$ & $2.66(2)$ & $\mathrm{Pb} 1-\mathrm{O} 5 \mathrm{~B}$ & $2.73(2)$ \\
\hline $\mathrm{Pb} 2-\mathrm{O} 12 \mathrm{C}$ & $2.41(2)$ & $\mathrm{Pb} 2-\mathrm{O} 10 \mathrm{~A}$ & $2.53(2)$ \\
\hline $\mathrm{Pb} 2-\mathrm{O} 7$ & $2.53(2)$ & $\mathrm{Pb} 2-\mathrm{O} 8$ & $2.655(19)$ \\
\hline $\mathrm{Pb} 2-\mathrm{O} 11 \mathrm{C}$ & $2.69(2)$ & $\mathrm{O} 4 \mathrm{~A}-\mathrm{Pb} 1-\mathrm{O} 1$ & $76.8(7)$ \\
\hline $\mathrm{O} 4 \mathrm{~A}-\mathrm{Pb} 1-\mathrm{O} 3 \mathrm{~A}$ & $50.1(7)$ & $\mathrm{O} 1-\mathrm{Pb} 1-\mathrm{O} 3 \mathrm{~A}$ & $126.5(7)$ \\
\hline $\mathrm{O} 4 \mathrm{~A}-\mathrm{Pb} 1-\mathrm{O} 6 \mathrm{~B}$ & $81.8(8)$ & $\mathrm{O} 1-\mathrm{Pb} 1-\mathrm{O} 6 \mathrm{~B}$ & $83.6(7)$ \\
\hline $\mathrm{O} 3 \mathrm{~A}-\mathrm{Pb} 1-\mathrm{O} 6 \mathrm{~B}$ & $82.7(8)$ & $\mathrm{O} 4 \mathrm{~A}-\mathrm{Pb} 1-\mathrm{O} 2$ & $123.2(7)$ \\
\hline $\mathrm{O} 1-\mathrm{Pb} 1-\mathrm{O} 2$ & $49.1(7)$ & $\mathrm{O} 3 \mathrm{~A}-\mathrm{Pb} 1-\mathrm{O} 2$ & $159.6(7)$ \\
\hline $\mathrm{O} 6 \mathrm{~B}-\mathrm{Pb} 1-\mathrm{O} 2$ & $77.1(7)$ & $\mathrm{O} 4 \mathrm{~A}-\mathrm{Pb} 1-\mathrm{O} 5 \mathrm{~B}$ & $119.0(8)$ \\
\hline $\mathrm{O} 1-\mathrm{Pb} 1-\mathrm{O} 5 \mathrm{~B}$ & $121.2(8)$ & $\mathrm{O} 3 \mathrm{~A}-\mathrm{Pb} 1-\mathrm{O} 5 \mathrm{~B}$ & $85.3(8)$ \\
\hline $\mathrm{O} 6 \mathrm{~B}-\mathrm{Pb} 1-\mathrm{O} 5 \mathrm{~B}$ & $49.1(6)$ & $\mathrm{O} 2-\mathrm{Pb} 1-\mathrm{O} 5 \mathrm{~B}$ & $83.4(8)$ \\
\hline $\mathrm{O} 12 \mathrm{C}-\mathrm{Pb} 2-\mathrm{O} 10 \mathrm{~A}$ & $81.4(7)$ & $\mathrm{O} 12 \mathrm{C}-\mathrm{Pb} 2-\mathrm{O} 7$ & $79.9(8)$ \\
\hline $\mathrm{O} 10 \mathrm{~A}-\mathrm{Pb} 2-\mathrm{O} 7$ & $74.2(6)$ & $\mathrm{O} 12 \mathrm{C}-\mathrm{Pb} 2-\mathrm{O} 8$ & $79.6(8)$ \\
\hline $\mathrm{O} 10 \mathrm{~A}-\mathrm{Pb} 2-\mathrm{O} 8$ & $125.2(6)$ & $\mathrm{O} 7-\mathrm{Pb} 2-\mathrm{O} 8$ & $52.0(6)$ \\
\hline $\mathrm{O} 12 \mathrm{C}-\mathrm{Pb} 2-\mathrm{O} 11 \mathrm{C}$ & $50.2(7)$ & $\mathrm{O} 10 \mathrm{~A}-\mathrm{Pb} 2-\mathrm{O} 11 \mathrm{C}$ & $121.8(6)$ \\
\hline $\mathrm{O} 7-\mathrm{Pb} 2-\mathrm{O} 11 \mathrm{C}$ & $117.6(7)$ & $\mathrm{O} 8-\mathrm{Pb} 2-\mathrm{O} 11 \mathrm{C}$ & $80.8(7)$ \\
\hline \multicolumn{4}{|c|}{3} \\
\hline $\mathrm{Cd} 1-\mathrm{O} 11 \mathrm{~A}$ & $2.268(10)$ & $\mathrm{Cd} 1-\mathrm{O} 7$ & $2.328(11)$ \\
\hline $\mathrm{Cd} 1-\mathrm{N} 2 \mathrm{~B}$ & $2.339(11)$ & $\mathrm{Cd} 1-\mathrm{O} 1$ & $2.351(10)$ \\
\hline $\mathrm{Cd} 1-\mathrm{O} 12 \mathrm{~B}$ & $2.368(10)$ & $\mathrm{Cd} 1-\mathrm{O} 2$ & $2.461(10)$ \\
\hline $\mathrm{Cd} 2-\mathrm{O} 4$ & $2.288(12)$ & $\mathrm{Cd} 2-\mathrm{O} 14$ & $2.289(14)$ \\
\hline $\mathrm{Cd} 2-\mathrm{O} 9$ & $2.297(10)$ & $\mathrm{Cd} 2-\mathrm{N} 1 \mathrm{C}$ & $2.302(12)$ \\
\hline
\end{tabular}




\begin{tabular}{|c|c|c|c|}
\hline $\mathrm{Cd} 2-\mathrm{O} 6 \mathrm{C}$ & $2.403(11)$ & $\mathrm{Cd} 2-\mathrm{O} 3$ & $2.444(11)$ \\
\hline $\mathrm{Cd} 2-\mathrm{O} 8$ & $2.645(12)$ & $\mathrm{Cd} 3-\mathrm{O} 5 \mathrm{D}$ & $2.164(12)$ \\
\hline $\mathrm{Cd} 3-\mathrm{O} 15$ & $2.230(13)$ & $\mathrm{Cd} 3-\mathrm{O} 13$ & $2.264(14)$ \\
\hline $\mathrm{Cd} 3-\mathrm{O} 16$ & $2.324(14)$ & $\mathrm{Cd} 3-\mathrm{O} 17$ & $2.381(13)$ \\
\hline $\mathrm{Cd} 3-\mathrm{O} 12$ & $2.647(9)$ & $\mathrm{Cd} 4-\mathrm{O} 20 \mathrm{C}$ & $2.268(11)$ \\
\hline $\mathrm{Cd} 4-\mathrm{O} 24$ & $2.339(11)$ & $\mathrm{Cd} 4-\mathrm{N} 3$ & $2.342(11)$ \\
\hline $\mathrm{Cd} 4-\mathrm{O} 28 \mathrm{E}$ & $2.362(12)$ & $\mathrm{Cd} 4-\mathrm{O} 18$ & $2.365(14)$ \\
\hline $\mathrm{Cd} 4-\mathrm{O} 27 \mathrm{E}$ & $2.457(13)$ & $\mathrm{Cd} 4-\mathrm{O} 22$ & $2.306(10)$ \\
\hline $\mathrm{Cd} 5-\mathrm{N} 4 \mathrm{C}$ & $2.338(13)$ & $\mathrm{Cd} 5-\mathrm{O} 25$ & $2.359(13)$ \\
\hline $\mathrm{Cd} 5-\mathrm{O} 31$ & $2.375(11)$ & $\mathrm{Cd} 5-\mathrm{O} 29 \mathrm{C}$ & $2.380(12)$ \\
\hline $\mathrm{Cd} 5-\mathrm{O} 26$ & $2.429(12)$ & $\mathrm{Cd} 5-\mathrm{O} 23$ & $2.549(11)$ \\
\hline $\mathrm{Cd} 6-\mathrm{O} 19 \mathrm{~F}$ & $2.131(16)$ & $\mathrm{Cd} 6-\mathrm{O} 34$ & $2.189(14)$ \\
\hline Cd6-O32 & $2.29(3)$ & $\mathrm{Cd} 6-\mathrm{O} 29$ & $2.334(10)$ \\
\hline $\mathrm{Cd} 6-\mathrm{O} 33$ & $2.41(2)$ & $\mathrm{Cd} 6-\mathrm{O} 35$ & $2.46(2)$ \\
\hline $\mathrm{O} 11 \mathrm{~A}-\mathrm{Cd} 1-\mathrm{O} 7$ & $89.1(5)$ & $\mathrm{O} 11 \mathrm{~A}-\mathrm{Cd} 1-\mathrm{N} 2 \mathrm{~B}$ & 141.9(3) \\
\hline $\mathrm{O} 7-\mathrm{Cd} 1-\mathrm{N} 2 \mathrm{~B}$ & $90.4(4)$ & $\mathrm{O} 11 \mathrm{~A}-\mathrm{Cd} 1-\mathrm{O} 1$ & $85.7(3)$ \\
\hline $\mathrm{O} 7-\mathrm{Cd} 1-\mathrm{O} 1$ & $90.4(4)$ & $\mathrm{N} 2 \mathrm{~B}-\mathrm{Cd} 1-\mathrm{O} 1$ & $132.5(4)$ \\
\hline $\mathrm{O} 11 \mathrm{~A}-\mathrm{Cd} 1-\mathrm{O} 12$ & $91.4(4)$ & $\mathrm{O} 7-\mathrm{Cd} 1-\mathrm{O} 12 \mathrm{~B}$ & $151.4(4)$ \\
\hline \multicolumn{4}{|l|}{ B } \\
\hline $\mathrm{N} 2 \mathrm{~B}-\mathrm{Cd} 1-\mathrm{O} 12 \mathrm{~B}$ & $72.0(4)$ & $\mathrm{O} 1-\mathrm{Cd} 1-\mathrm{O} 12 \mathrm{~B}$ & 118.2(4) \\
\hline $\mathrm{O} 11 \mathrm{~A}-\mathrm{Cd} 1-\mathrm{O} 2$ & $138.9(3)$ & $\mathrm{O} 7-\mathrm{Cd} 1-\mathrm{O} 2$ & $86.2(4)$ \\
\hline $\mathrm{N} 2 \mathrm{~B}-\mathrm{Cd} 1-\mathrm{O} 2$ & $79.0(4)$ & $\mathrm{O} 1-\mathrm{Cd} 1-\mathrm{O} 2$ & $53.6(3)$ \\
\hline $\mathrm{O} 12 \mathrm{~B}-\mathrm{Cd} 1-\mathrm{O} 2$ & $111.3(4)$ & $\mathrm{O} 4-\mathrm{Cd} 2-\mathrm{O} 14$ & $92.4(5)$ \\
\hline $\mathrm{O} 4-\mathrm{Cd} 2-\mathrm{O} 9$ & $83.9(4)$ & $\mathrm{O} 14-\mathrm{Cd} 2-\mathrm{O} 9$ & $90.8(6)$ \\
\hline $\mathrm{O} 4-\mathrm{Cd} 2-\mathrm{N} 1 \mathrm{C}$ & $143.2(4)$ & $\mathrm{O} 14-\mathrm{Cd} 2-\mathrm{N} 1 \mathrm{C}$ & $95.6(5)$ \\
\hline $\mathrm{O} 9-\mathrm{Cd} 2-\mathrm{N} 1 \mathrm{C}$ & $131.7(4)$ & $\mathrm{O} 4-\mathrm{Cd} 2-\mathrm{O} 6 \mathrm{C}$ & $110.1(5)$ \\
\hline $\mathrm{O} 14-\mathrm{Cd} 2-\mathrm{O} 6 \mathrm{C}$ & $155.2(5)$ & $\mathrm{O} 9-\mathrm{Cd} 2-\mathrm{O} 6 \mathrm{C}$ & $81.7(4)$ \\
\hline $\mathrm{N} 1 \mathrm{C}-\mathrm{Cd} 2-\mathrm{O} 6 \mathrm{C}$ & $72.9(4)$ & $\mathrm{O} 4-\mathrm{Cd} 2-\mathrm{O} 3$ & $55.0(4)$ \\
\hline $\mathrm{O} 14-\mathrm{Cd} 2-\mathrm{O} 3$ & $93.6(6)$ & $\mathrm{O} 9-\mathrm{Cd} 2-\mathrm{O} 3$ & $138.8(4)$ \\
\hline
\end{tabular}




\begin{tabular}{|c|c|c|c|}
\hline $\mathrm{N} 1 \mathrm{C}-\mathrm{Cd} 2-\mathrm{O} 3$ & $88.6(4)$ & $\mathrm{O} 6 \mathrm{C}-\mathrm{Cd} 2-\mathrm{O} 3$ & $107.7(5)$ \\
\hline $\mathrm{O} 4-\mathrm{Cd} 2-\mathrm{O} 8$ & $134.9(4)$ & $\mathrm{O} 14-\mathrm{Cd} 2-\mathrm{O} 8$ & $79.6(5)$ \\
\hline $\mathrm{O} 9-\mathrm{Cd} 2-\mathrm{O} 8$ & $52.3(4)$ & $\mathrm{N} 1 \mathrm{C}-\mathrm{Cd} 2-\mathrm{O} 8$ & $81.9(4)$ \\
\hline $\mathrm{O} 6 \mathrm{C}-\mathrm{Cd} 2-\mathrm{O} 8$ & $77.0(4)$ & $\mathrm{O} 3-\mathrm{Cd} 2-\mathrm{O} 8$ & $167.7(4)$ \\
\hline $\mathrm{O} 5 \mathrm{D}-\mathrm{Cd} 3-\mathrm{O} 15$ & $123.5(6)$ & $\mathrm{O} 5 \mathrm{D}-\mathrm{Cd} 3-\mathrm{O} 13$ & $117.8(5)$ \\
\hline $\mathrm{O} 15-\mathrm{Cd} 3-\mathrm{O} 13$ & $88.3(7)$ & $\mathrm{O} 5 \mathrm{D}-\mathrm{Cd} 3-\mathrm{O} 16$ & $137.2(5)$ \\
\hline $\mathrm{O} 15-\mathrm{Cd} 3-\mathrm{O} 16$ & $91.7(6)$ & $\mathrm{O} 13-\mathrm{Cd} 3-\mathrm{O} 16$ & $83.2(5)$ \\
\hline $\mathrm{O} 5 \mathrm{D}-\mathrm{Cd} 3-\mathrm{O} 17$ & $81.9(5)$ & $\mathrm{O} 15-\mathrm{Cd} 3-\mathrm{O} 17$ & $83.6(7)$ \\
\hline $\mathrm{O} 13-\mathrm{Cd} 3-\mathrm{O} 17$ & $159.7(5)$ & $\mathrm{O} 16-\mathrm{Cd} 3-\mathrm{O} 17$ & $78.5(6)$ \\
\hline $\mathrm{O} 5 \mathrm{D}-\mathrm{Cd} 3-\mathrm{O} 12$ & $82.7(4)$ & $\mathrm{O} 15-\mathrm{Cd} 3-\mathrm{O} 12$ & $75.7(4)$ \\
\hline $\mathrm{O} 13-\mathrm{Cd} 3-\mathrm{O} 12$ & $52.5(3)$ & $\mathrm{O} 16-\mathrm{Cd} 3-\mathrm{O} 12$ & $133.6(4)$ \\
\hline $\mathrm{O} 17-\mathrm{Cd} 3-\mathrm{O} 12$ & $141.3(5)$ & $\mathrm{O} 20 \mathrm{C}-\mathrm{Cd} 4-\mathrm{O} 24$ & $84.2(4)$ \\
\hline $\mathrm{O} 20 \mathrm{C}-\mathrm{Cd} 4-\mathrm{N} 3$ & $141.6(4)$ & $\mathrm{O} 24-\mathrm{Cd} 4-\mathrm{N} 3$ & $94.2(4)$ \\
\hline $\mathrm{O} 20 \mathrm{C}-\mathrm{Cd} 4-\mathrm{O} 28 \mathrm{E}$ & $136.8(4)$ & $\mathrm{O} 24-\mathrm{Cd} 4-\mathrm{O} 28 \mathrm{E}$ & $89.8(5)$ \\
\hline $\mathrm{N} 3-\mathrm{Cd} 4-\mathrm{O} 28 \mathrm{E}$ & $81.4(4)$ & $\mathrm{O} 20 \mathrm{C}-\mathrm{Cd} 4-\mathrm{O} 18$ & $94.2(5)$ \\
\hline $\mathrm{O} 24-\mathrm{Cd} 4-\mathrm{O} 18$ & $155.2(4)$ & $\mathrm{N} 3-\mathrm{Cd} 4-\mathrm{O} 18$ & $71.8(4)$ \\
\hline $\mathrm{O} 28 \mathrm{E}-\mathrm{Cd} 4-\mathrm{O} 18$ & $107.6(5)$ & $\mathrm{O} 20 \mathrm{C}-\mathrm{Cd} 4-\mathrm{O} 27 \mathrm{E}$ & $84.3(4)$ \\
\hline $\mathrm{O} 24-\mathrm{Cd} 4-\mathrm{O} 27 \mathrm{E}$ & $98.0(5)$ & $\mathrm{N} 3-\mathrm{Cd} 4-\mathrm{O} 27 \mathrm{E}$ & $133.6(4)$ \\
\hline $\mathrm{O} 28 \mathrm{E}-\mathrm{Cd} 4-\mathrm{O} 27 \mathrm{E}$ & $54.2(4)$ & $\mathrm{O} 18-\mathrm{Cd} 4-\mathrm{O} 27 \mathrm{E}$ & $106.4(5)$ \\
\hline $\mathrm{O} 22-\mathrm{Cd} 5-\mathrm{N} 4 \mathrm{C}$ & $135.8(4)$ & $\mathrm{O} 22-\mathrm{Cd} 5-\mathrm{O} 25$ & $87.1(4)$ \\
\hline $\mathrm{N} 4 \mathrm{C}-\mathrm{Cd} 5-\mathrm{O} 25$ & $134.1(5)$ & $\mathrm{O} 22-\mathrm{Cd} 5-\mathrm{O} 31$ & $84.7(4)$ \\
\hline $\mathrm{O} 4 \mathrm{C}-\mathrm{Cd} 5-\mathrm{O} 31$ & $97.0(5)$ & $\mathrm{O} 25-\mathrm{Cd} 5-\mathrm{O} 31$ & $103.8(5)$ \\
\hline $\mathrm{O} 22-\mathrm{Cd} 5-\mathrm{O} 29 \mathrm{C}$ & $88.8(4)$ & $\mathrm{N} 4 \mathrm{C}-\mathrm{Cd} 5-\mathrm{O} 29 \mathrm{C}$ & $73.4(4)$ \\
\hline $\mathrm{O} 25-\mathrm{Cd} 5-\mathrm{O} 29 \mathrm{C}$ & $96.8(5)$ & $\mathrm{O} 31-\mathrm{Cd} 5-\mathrm{O} 29 \mathrm{C}$ & $158.0(5)$ \\
\hline $\mathrm{O} 22-\mathrm{Cd} 5-\mathrm{O} 26$ & $132.1(4)$ & $\mathrm{N} 4 \mathrm{C}-\mathrm{Cd} 5-\mathrm{O} 26$ & $91.2(4)$ \\
\hline $\mathrm{O} 25-\mathrm{Cd} 5-\mathrm{O} 26$ & $53.9(4)$ & $\mathrm{O} 31-\mathrm{Cd} 5-\mathrm{O} 26$ & $80.1(4)$ \\
\hline $\mathrm{O} 29 \mathrm{C}-\mathrm{Cd} 5-\mathrm{O} 26$ & 119.1(4) & $\mathrm{O} 22-\mathrm{Cd} 5-\mathrm{O} 23$ & $53.7(4)$ \\
\hline $\mathrm{N} 4 \mathrm{C}-\mathrm{Cd} 5-\mathrm{O} 23$ & $82.9(4)$ & $\mathrm{O} 25-\mathrm{Cd} 5-\mathrm{O} 23$ & $140.3(4)$ \\
\hline $\mathrm{O} 31-\mathrm{Cd} 5-\mathrm{O} 23$ & $80.5(4)$ & $\mathrm{O} 29 \mathrm{C}-\mathrm{Cd} 5-\mathrm{O} 23$ & $78.7(5)$ \\
\hline
\end{tabular}




\begin{tabular}{|c|c|c|c|}
\hline $\mathrm{O} 26-\mathrm{Cd} 5-\mathrm{O} 23$ & $158.8(4)$ & $\mathrm{O} 19 \mathrm{~F}-\mathrm{Cd} 6-\mathrm{O} 34$ & $94.3(13)$ \\
\hline $\mathrm{O} 19 \mathrm{~F}-\mathrm{Cd} 6-\mathrm{O} 32$ & $160.8(10)$ & $\mathrm{O} 34-\mathrm{Cd} 6-\mathrm{O} 32$ & $94.6(14)$ \\
\hline O19F-Cd6-O29 & $94.6(5)$ & $\mathrm{O} 34-\mathrm{Cd} 6-\mathrm{O} 29$ & $110.8(11)$ \\
\hline $\mathrm{O} 32-\mathrm{Cd} 6-\mathrm{O} 29$ & $98.1(8)$ & $\mathrm{O} 19 \mathrm{~F}-\mathrm{Cd} 6-\mathrm{O} 33$ & $79.7(8)$ \\
\hline $\mathrm{O} 34-\mathrm{Cd} 6-\mathrm{O} 33$ & $88.0(12)$ & $\mathrm{O} 32-\mathrm{Cd} 6-\mathrm{O} 33$ & $83.6(10)$ \\
\hline $\mathrm{O} 29-\mathrm{Cd} 6-\mathrm{O} 33$ & $160.9(6)$ & $\mathrm{O} 19 \mathrm{~F}-\mathrm{Cd} 6-\mathrm{O} 35$ & $84.6(8)$ \\
\hline O34-Cd6-O35 & $163.9(12)$ & $\mathrm{O} 32-\mathrm{Cd} 6-\mathrm{O} 35$ & $82.2(10)$ \\
\hline $\mathrm{O} 29-\mathrm{Cd} 6-\mathrm{O} 35$ & $85.3(6)$ & $\mathrm{O} 33-\mathrm{Cd} 6-\mathrm{O} 35$ & $76.0(7)$ \\
\hline \multicolumn{4}{|c|}{4} \\
\hline $\mathrm{Pb} 1-\mathrm{O} 5$ & $2.462(7)$ & $\mathrm{Pb} 1-\mathrm{O} 3 \mathrm{~A}$ & $2.509(6)$ \\
\hline $\mathrm{Pb} 1-\mathrm{O} 4 \mathrm{~A}$ & $2.558(7)$ & $\mathrm{Pb} 1-\mathrm{O} 1 \mathrm{~B}$ & $2.575(7)$ \\
\hline $\mathrm{Pb} 1-\mathrm{O} 2 \mathrm{C}$ & $2.692(6)$ & $\mathrm{O} 5-\mathrm{Pb} 1-\mathrm{O} 3 \mathrm{~A}$ & $103.3(2)$ \\
\hline $\mathrm{O} 5-\mathrm{Pb} 1-\mathrm{O} 4 \mathrm{~A}$ & $80.4(3)$ & $\mathrm{O} 3 \mathrm{~A}-\mathrm{Pb} 1-\mathrm{O} 4 \mathrm{~A}$ & $50.9(2)$ \\
\hline $\mathrm{O} 5-\mathrm{Pb} 1-\mathrm{O} 1 \mathrm{~B}$ & $124.1(3)$ & $\mathrm{O} 3 \mathrm{~A}-\mathrm{Pb} 1-\mathrm{O} 1 \mathrm{~B}$ & $95.3(3)$ \\
\hline $\mathrm{O} 4 \mathrm{~A}-\mathrm{Pb} 1-\mathrm{O} 1 \mathrm{~B}$ & $71.9(3)$ & $\mathrm{O} 5-\mathrm{Pb} 1-\mathrm{O} 2 \mathrm{C}$ & $68.5(2)$ \\
\hline $\mathrm{O} 3 \mathrm{~A}-\mathrm{Pb} 1-\mathrm{O} 2 \mathrm{C}$ & $73.7(2)$ & $\mathrm{O} 4 \mathrm{~A}-\mathrm{Pb} 1-\mathrm{O} 2 \mathrm{C}$ & $106.4(2)$ \\
\hline $\mathrm{O} 1 \mathrm{~B}-\mathrm{Pb} 1-\mathrm{O} 2 \mathrm{C}$ & $165.6(2)$ & & \\
\hline \multicolumn{4}{|c|}{ Symmetry codes: (A) $x, y+1, z$; (B) $x-1 / 2, y+1 / 2, z$; (C) $x, y-1, z$; (D) $x+$} \\
\hline \multicolumn{4}{|c|}{$1 / 2, y-1 / 2, z$ for 1. (A) $x+1, y, z$; (B) $x, y-1, z$; (C) $x+1, y+1, z$; (D) $x-1, y$, } \\
\hline \multicolumn{4}{|c|}{$z$; (E) $x, y+1, z$; (F) $x-1, y-1, z$ for 2. (A) $x+1, y, z$; (B) $x+1, y-1, z$; (C) $x$} \\
\hline \multicolumn{4}{|c|}{$y+1, z ;(\mathrm{D})-x+1, y+3 / 2,-z+1 ; \quad$ (E) $x+1, y+1, z ;(\mathrm{F})-x+2, y-3 / 2,-z$} \\
\hline \multicolumn{4}{|c|}{$+2(\mathrm{G})-x+1, y-3 / 2,-z+1 ;(\mathrm{H}) x, y-1, z$; (I) $x-1, y, z$; (J) $x-1, y+1, z$; } \\
\hline \multicolumn{4}{|c|}{ (K) $-x+2, y+3 / 2,-z+2$; (L) $x-1, y-1, z$ for 3. (A) $-x, y+1 / 2,-z+1 / 2$} \\
\hline \multicolumn{4}{|c|}{ (B) $x+1 / 2,-y+3 / 2,-z+1$; (C) $x+1, y, z$; (D) $x-1 / 2,-y+3 / 2,-z+1$; (E) } \\
\hline \multicolumn{4}{|c|}{$x-1, y, z ;(\mathrm{F})-x, y-1 / 2,-z+1 / 2$ for 4} \\
\hline
\end{tabular}




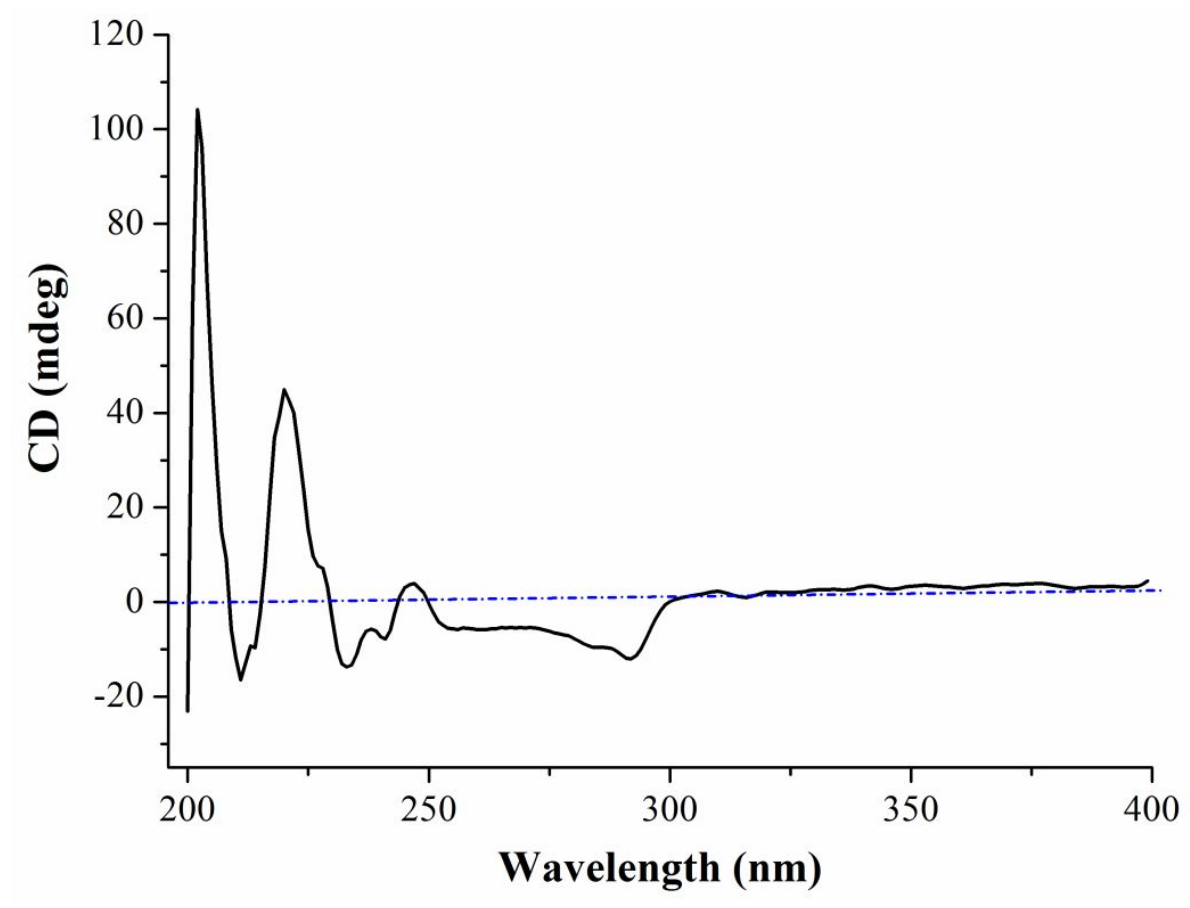

Figure S1. Solid-state $\mathrm{CD}$ spectra of $\mathrm{H}_{3} \mathrm{~L}^{2}$ ligand in $\mathrm{KBr}$.

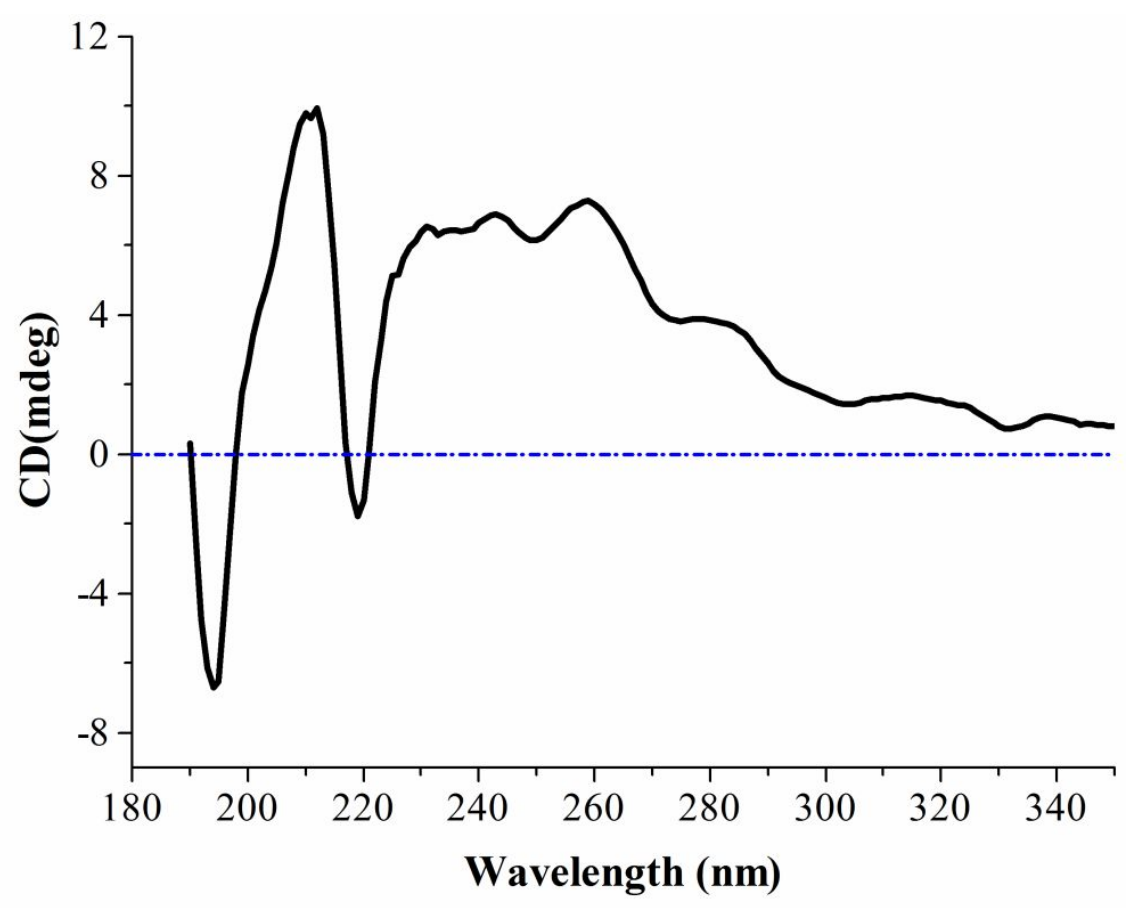

Figure S2. Solid-state $\mathrm{CD}$ spectra of $\mathrm{H}_{3} \mathrm{~L}^{3}$ ligand in $\mathrm{KBr}$. 


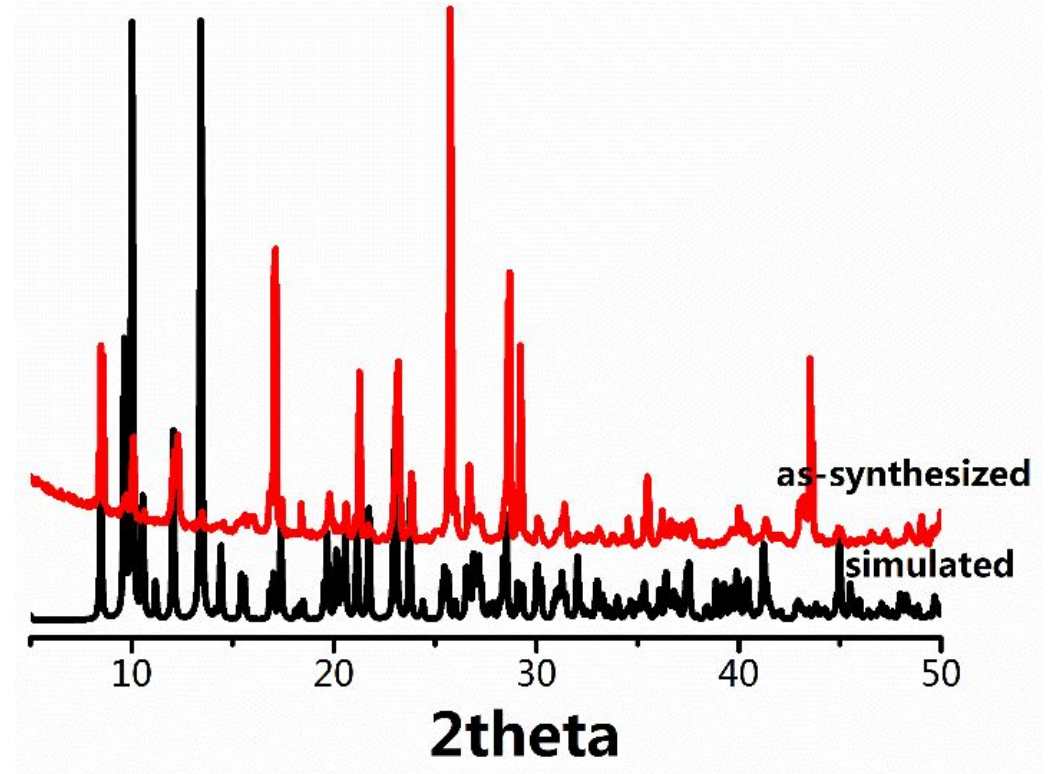

Figure S3. As-synthesized and simulated PXRD patterns of compound $\mathbf{1 .}$

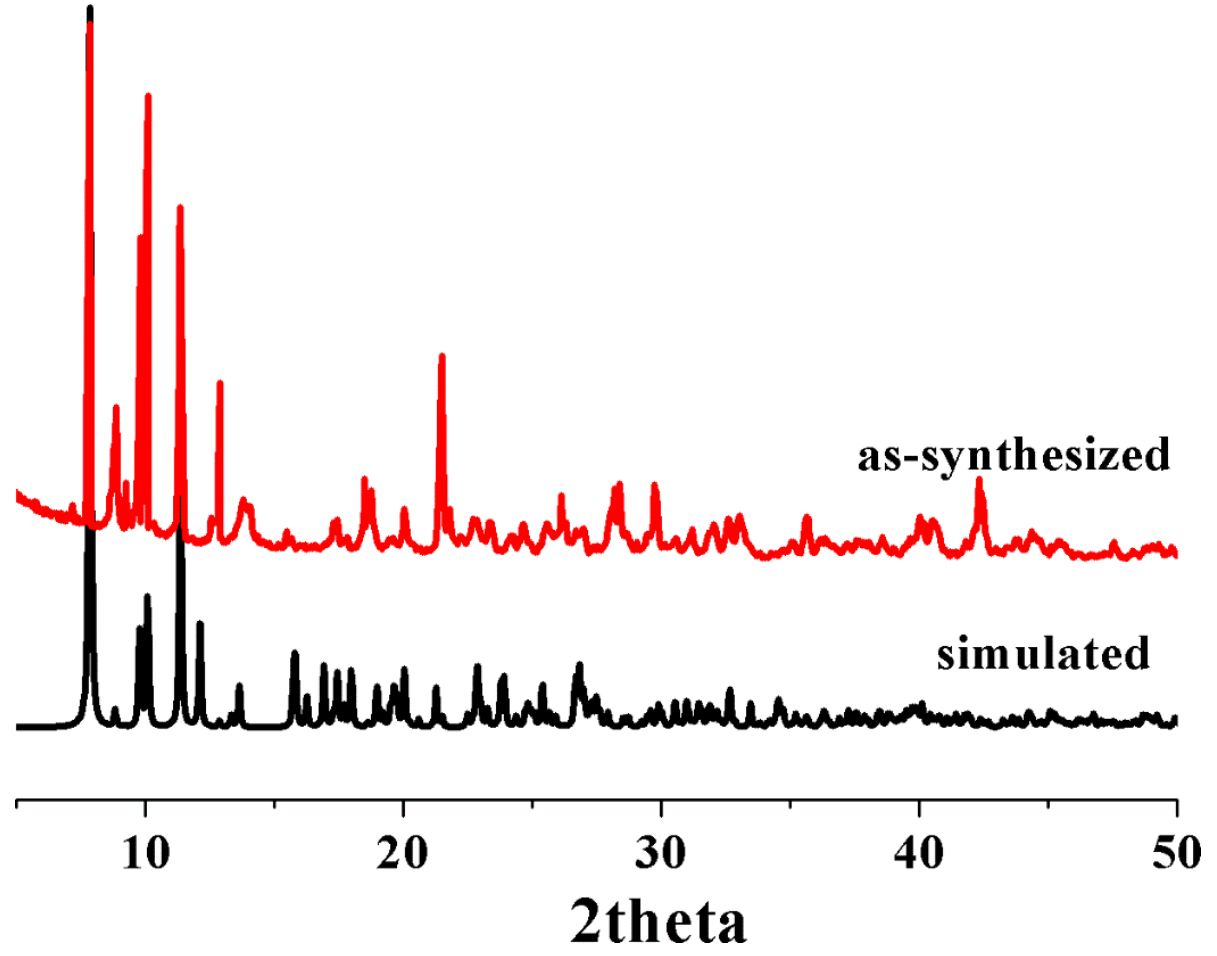


Figure S4. As-synthesized and simulated PXRD patterns of compound 2.

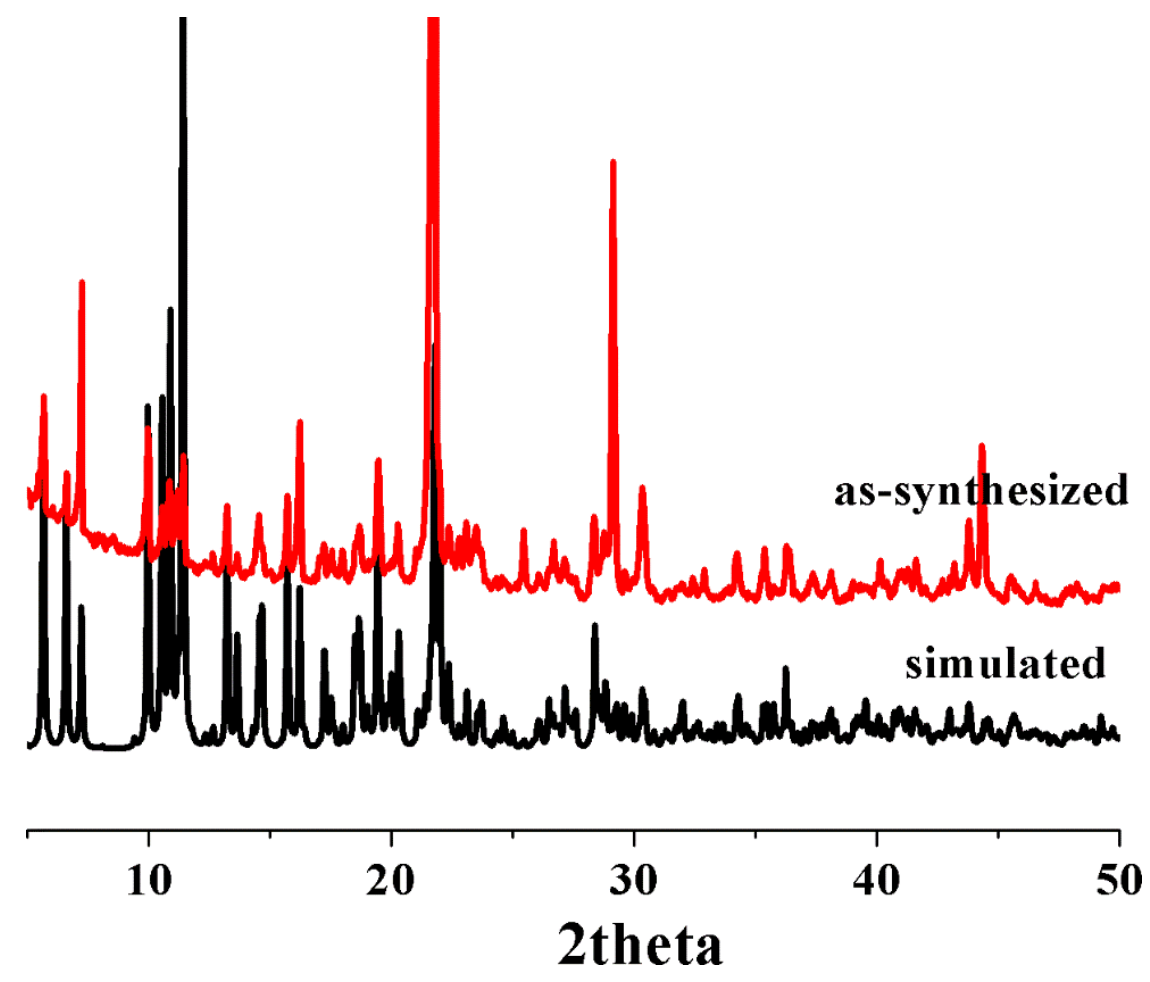

Figure S5. As-synthesized and simulated PXRD patterns of compound 3.

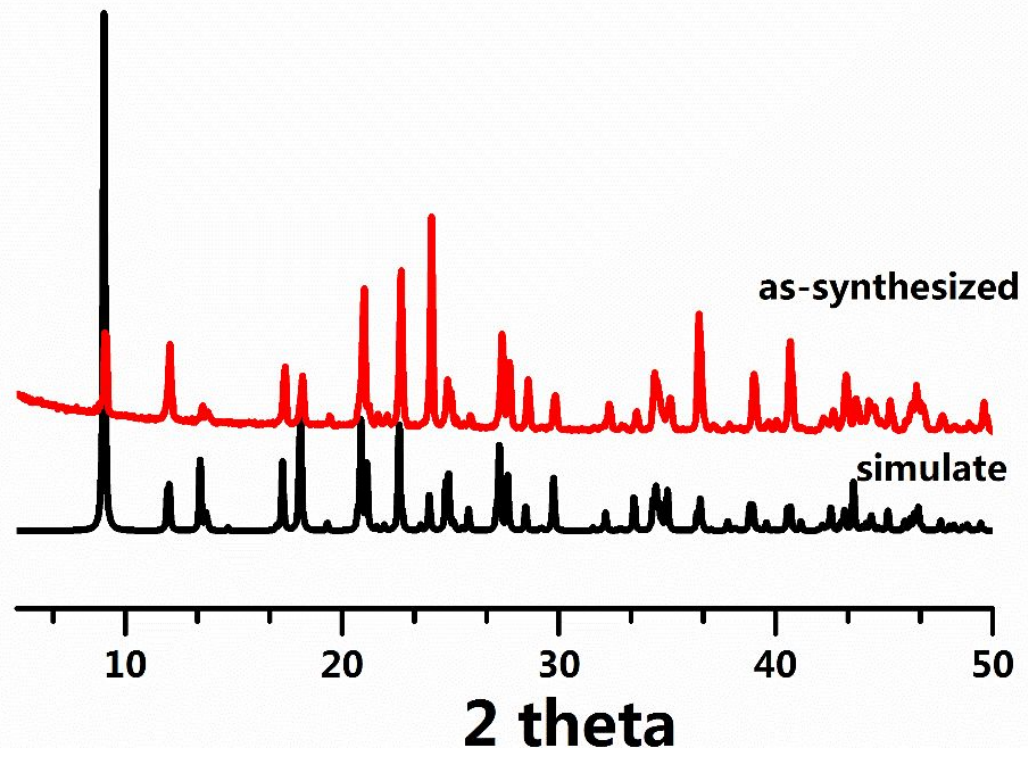

Figure S6. As-synthesized and simulated PXRD patterns of compound 4. 


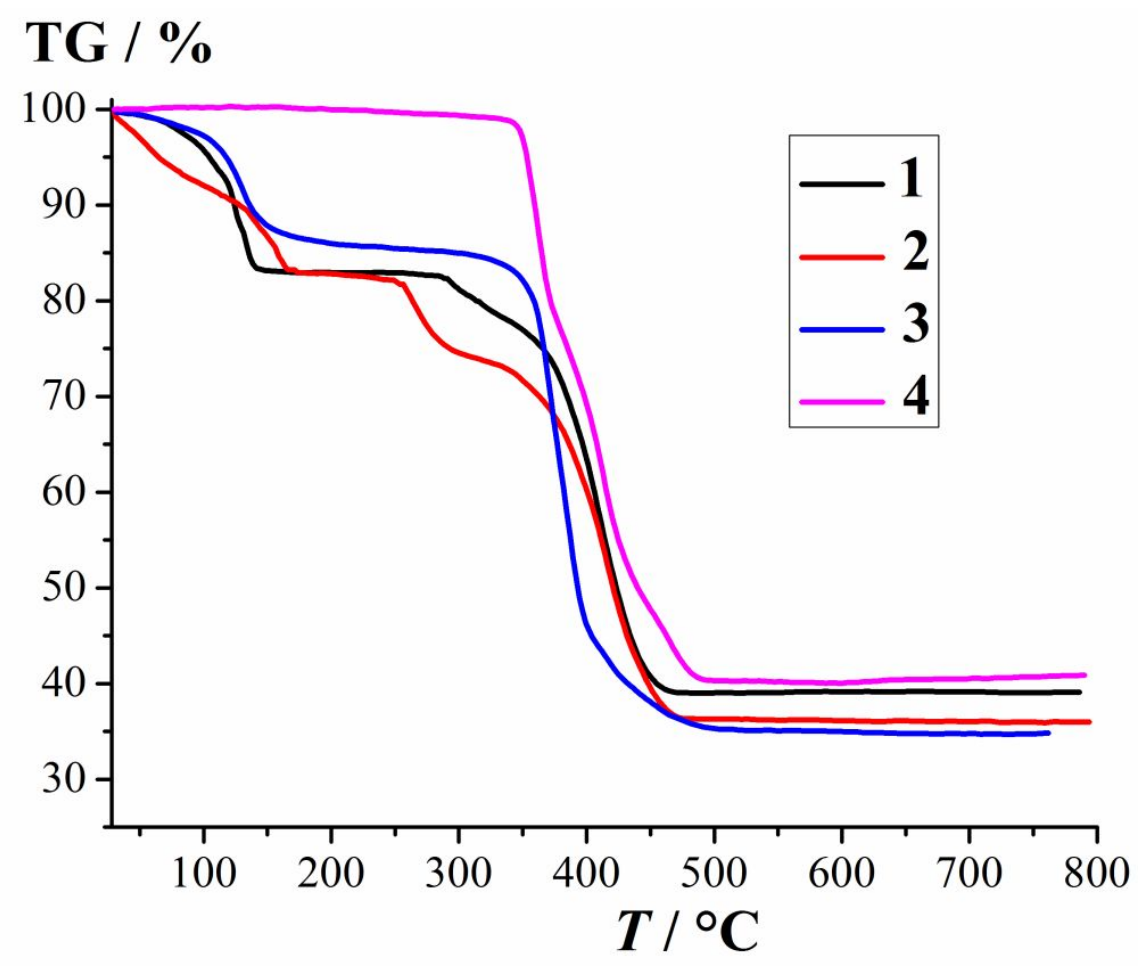

Figure S7. TGA curves of compounds 1-4.

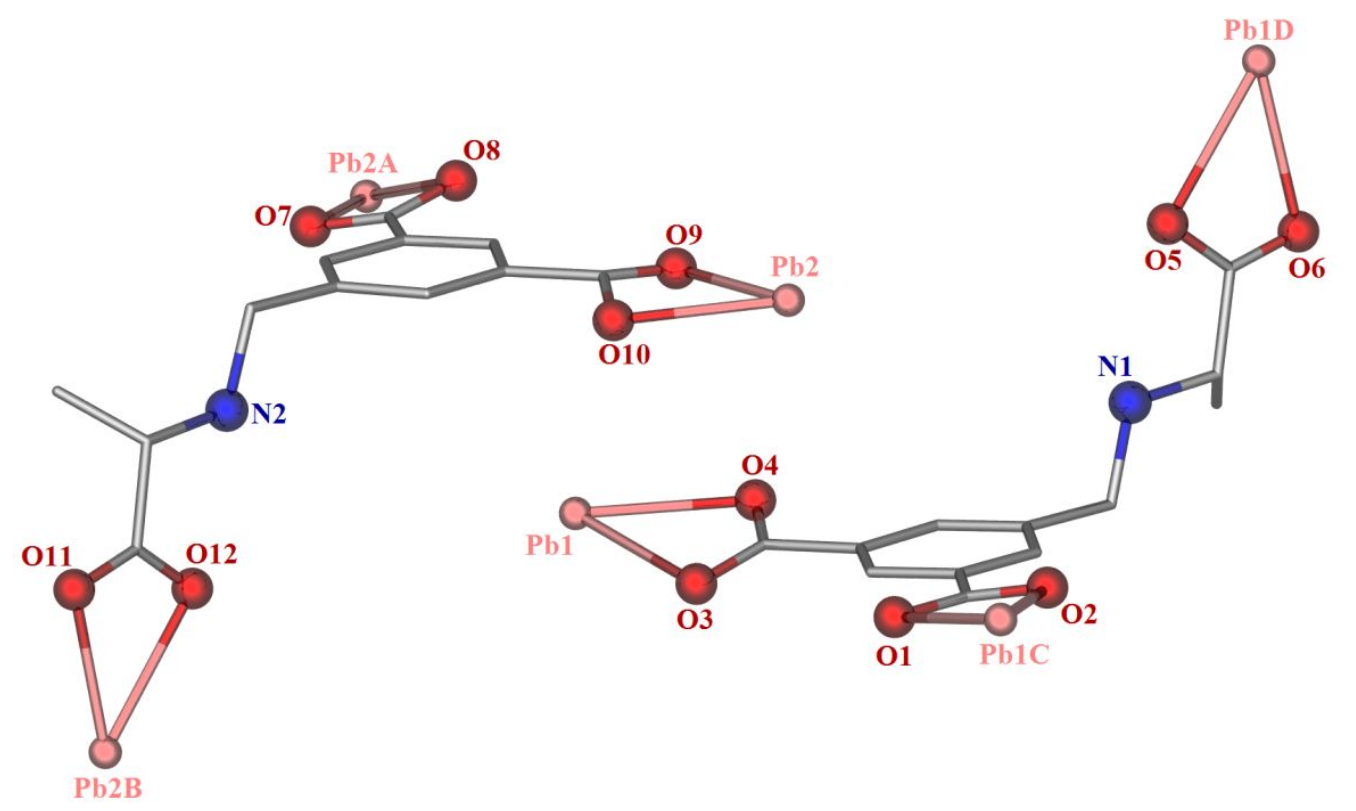

Figure S8. View of two independent zwitterionic $\left(\mathrm{HL}^{2}\right)^{2-}$ ligands acting as $\kappa_{6^{-}}$and $\kappa_{5}$-linkers to connect three symmetry-related $\mathrm{Pb} 1$ and $\mathrm{Pb} 2$ in $\mathbf{1}$, respectively. Symmetry code: (A) $x, 1+y, z$; (B) $-1 / 2+x, 1 / 2+y, z$; (C) $x,-1+y, z$; (D) $1 / 2+x$, 


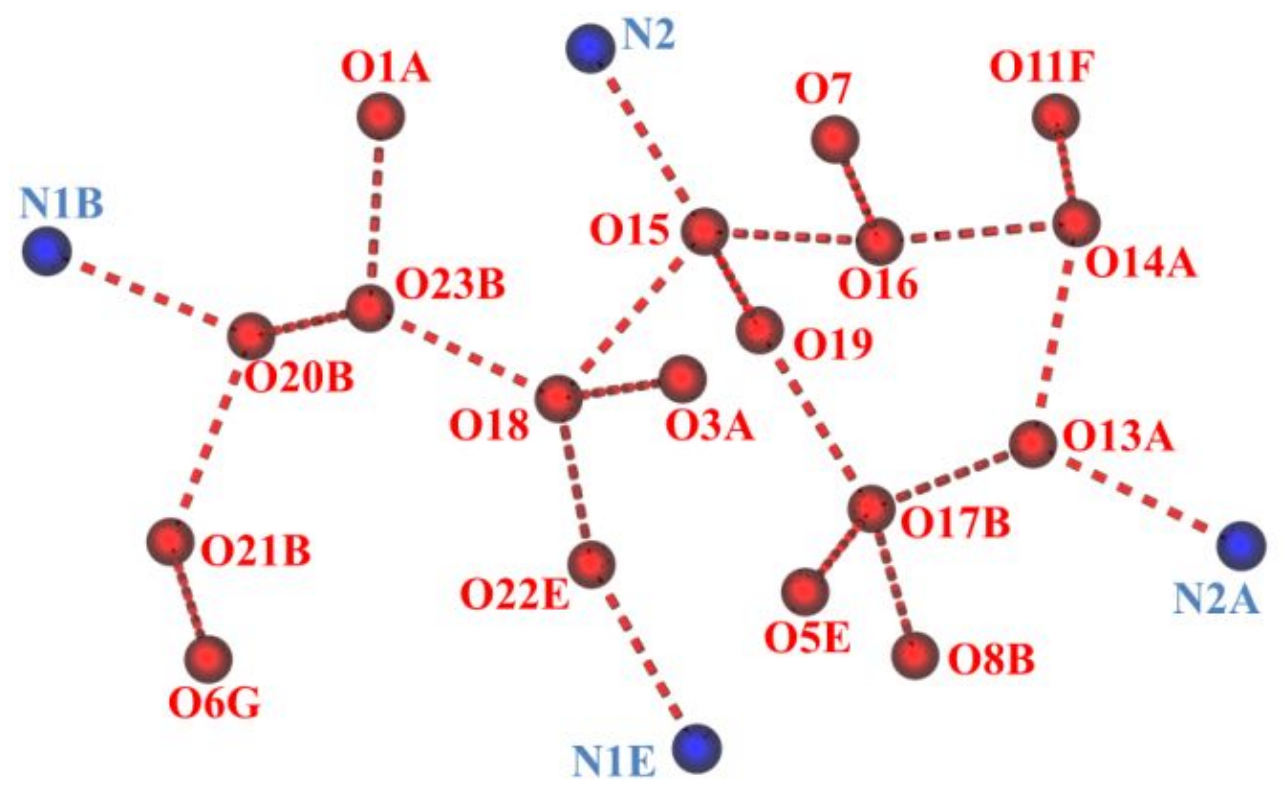

Figure S9. Water cluster $\left(\mathrm{H}_{2} \mathrm{O}\right)_{11}$ formed through hydrogen-bonding interactions between lattice water molecules O13A, O14A, O15, O16, O17B, O18, O19, O20B, $\mathrm{O} 21 \mathrm{~B}, \mathrm{O} 22 \mathrm{E}$, and $\mathrm{O} 23 \mathrm{~B}(\mathrm{O} 18 \cdots \mathrm{O} 22 \mathrm{E}=2.651 \AA, \mathrm{O} 15 \cdots \mathrm{O} 18=2.816 \AA, \mathrm{O} 15 \cdots \mathrm{O} 19$ $=2.688 \AA, \mathrm{O} 23 \mathrm{~B} \cdots \mathrm{O} 18=2.567 \AA, \mathrm{O} 16 \cdots \mathrm{O} 15=2.622 \AA, \mathrm{O} 14 \mathrm{~A} \cdots \mathrm{O} 16=2.817 \AA$, $\mathrm{O} 13 \mathrm{~A} \cdots \mathrm{O} 14 \mathrm{~A}=2.642 \AA, \mathrm{O} 13 \mathrm{~A} \cdots \mathrm{O} 17 \mathrm{~B}=2.783 \AA, \mathrm{O} 20 \mathrm{~B} \cdots \mathrm{O} 23 \mathrm{~B}=2.669 \AA$, $\mathrm{O} 20 \mathrm{~B} \cdots \mathrm{O} 21 \mathrm{~B}=2.858 \AA$ ) in $\mathbf{1}$. Hydrogen-bonding connections between water cluster $\left(\mathrm{H}_{2} \mathrm{O}\right)_{11}$ and $\mathrm{N}_{\text {amino }}$ and $\mathrm{O}_{\text {carboxylate }}$ of ligands $\left(\mathrm{HL}^{1}\right)^{2-}(\mathrm{N} 1 \mathrm{E} \cdots \mathrm{O} 22 \mathrm{E}=2.798 \AA$, $\mathrm{O} 18 \cdots \mathrm{O} 3 \mathrm{~A}=2.879 \AA, \mathrm{N} 2 \cdots \mathrm{O} 15=2.775 \AA, \mathrm{O} 16 \cdots \mathrm{O} 7=2.740 \AA, \mathrm{O} 14 \mathrm{~A} \cdots \mathrm{O} 11 \mathrm{~F}=$ $2.935 \AA, \mathrm{N} 2 \mathrm{~A} \cdots \mathrm{O} 13 \mathrm{~A}=2.871 \AA, \mathrm{O} 21 \mathrm{~B} \cdots \mathrm{O} 6 \mathrm{G}=2.731 \AA, \mathrm{O} 17 \mathrm{~B} \cdots \mathrm{O} 5 \mathrm{E}=2.658 \AA$, $\mathrm{N} 1 \mathrm{~B} \cdots \mathrm{O} 20 \mathrm{~B}=2.756 \AA, \mathrm{O} 17 \mathrm{~B} \cdots \mathrm{O} 8 \mathrm{~B}=2.704 \AA, \mathrm{O} 23 \mathrm{~B} \cdots \mathrm{O} 1 \mathrm{~A}=2.599 \AA)$. Hydrogen bond lengths calculated by Mercury program. Symmetry code: (A) $x, 1+y, z$; (B) $-1 / 2+x, 1 / 2+y, z ;(\mathrm{E})-1 / 2+x, 3 / 2+y, z ;(\mathrm{F})-1 / 2+x, 1 / 2+y,-2+z ;(\mathrm{G}) 1-x, 1$ $+y, 1-z$. 


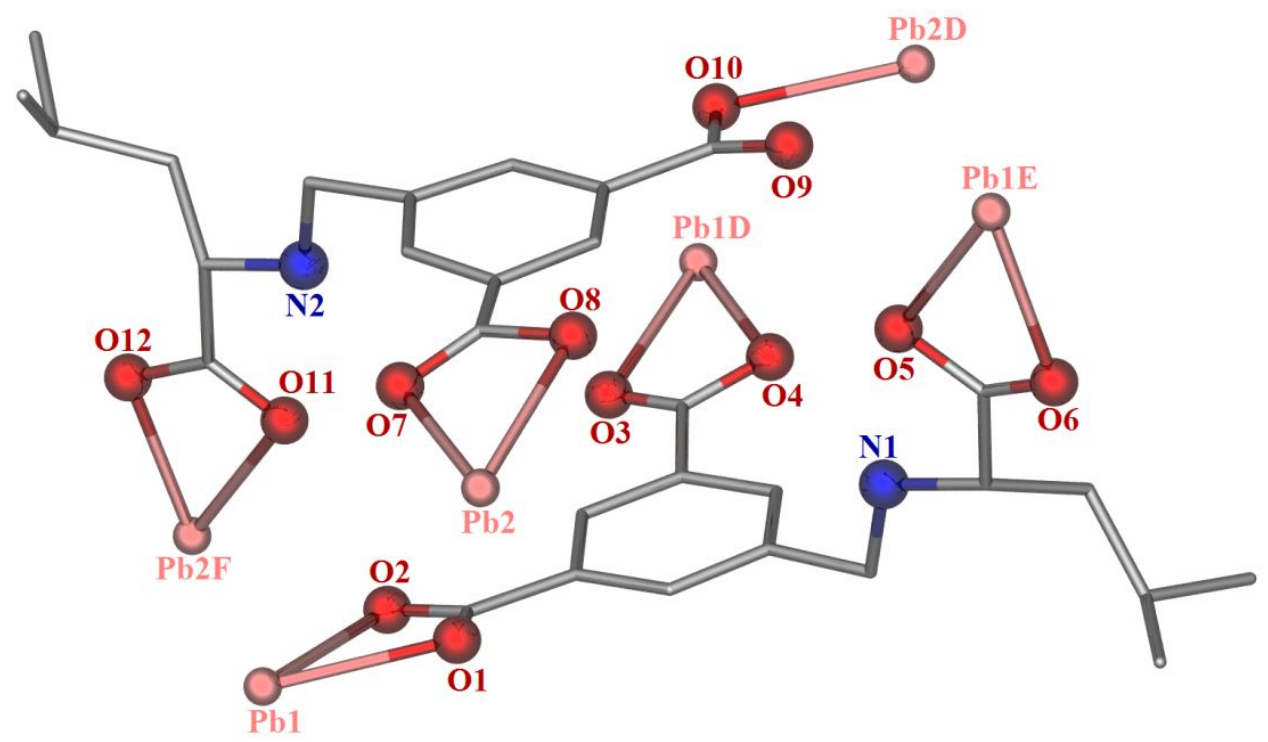

Figure S10. View of two independent zwitterionic $\left(\mathrm{HL}^{1}\right)^{2-}$ ligands chelating three symmetry-related $\mathrm{Pb} 1$ and three symmetry-related $\mathrm{Pb} 2$ in $\mathbf{2}$, respectively. Symmetry code: (D) $-1+x, y, z$; (E) $x, 1+y, z ;(\mathrm{F})-1+x,-1+y, z$.
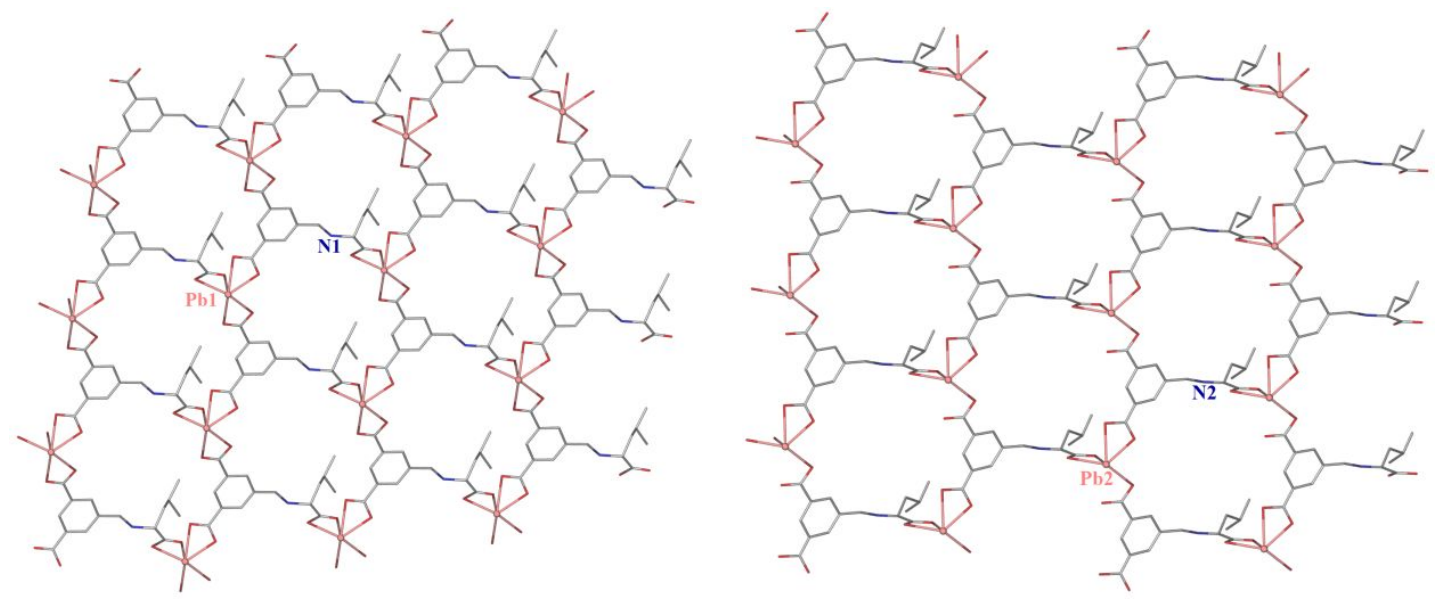

Figure S11. View of two types of unsupported wave-like homochiral polymeric layers with $6^{3}$ topologies in 2 , namely, $\mathrm{Pb} 1$-layer being fabricated by the coordination of the symmetry-related $\left[\left(\mathrm{HL}^{2}\right)_{\text {including } \mathrm{N} 1}\right]^{2-}$ ligands and symmetry-related $\mathrm{Pb} 1$ nodes, and $\mathrm{Pb}$-layer being created by the coordination of the symmetry-related $\left[\left(\mathrm{HL}^{2}\right)_{\text {including }}\right.$ $\left.{ }_{\mathrm{N}} 2\right]^{2-}$ ligands and symmetry-related $\mathrm{Pb} 2$ nodes. 


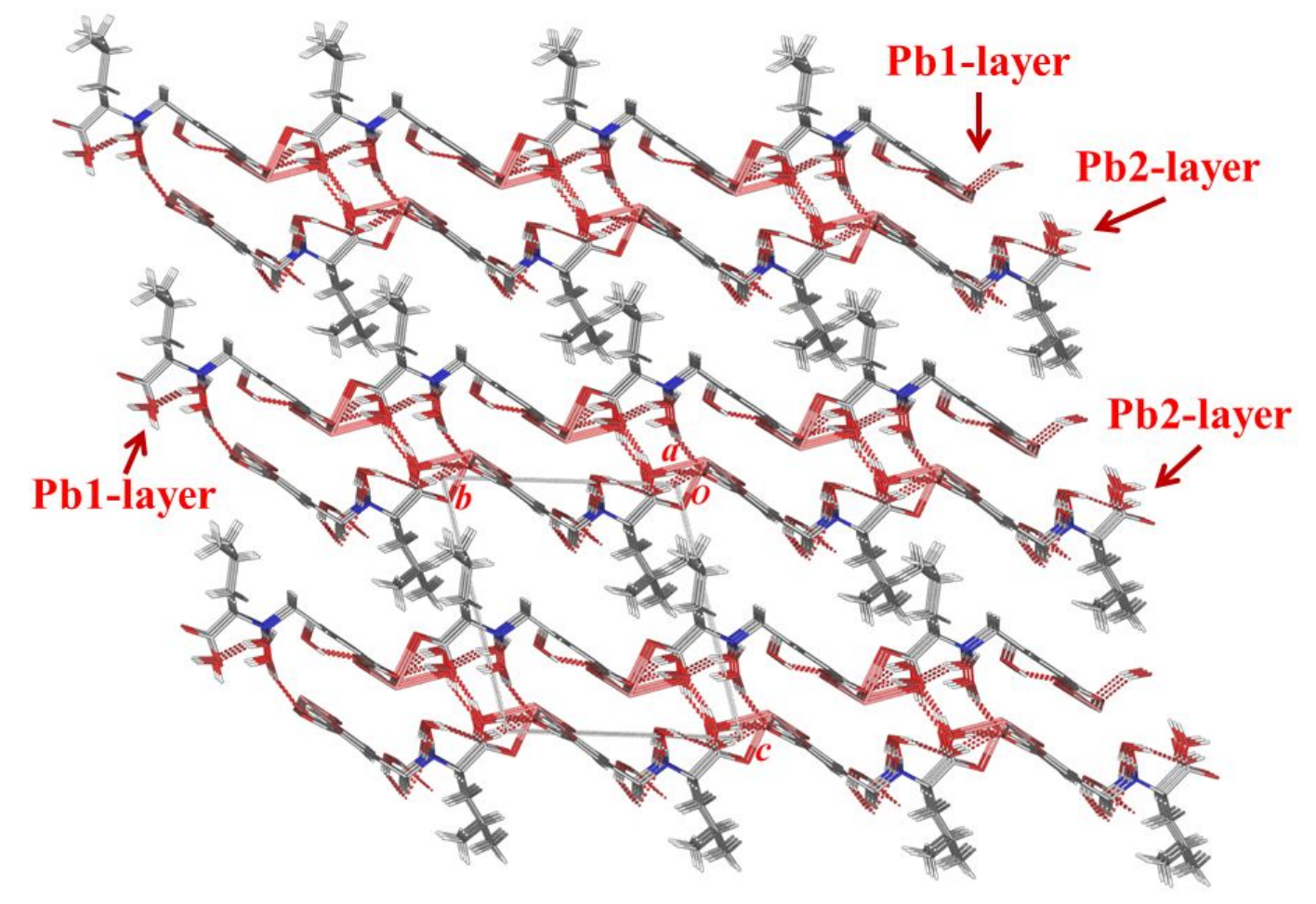

Figure S12. Interdigitation of isobutyl groups between two bilayers in 2, showing the stacking of $\mathrm{Pb} 1$-layer and $\mathrm{Pb}$ 2-layer following the $\mathrm{ABAB}$ pattern.

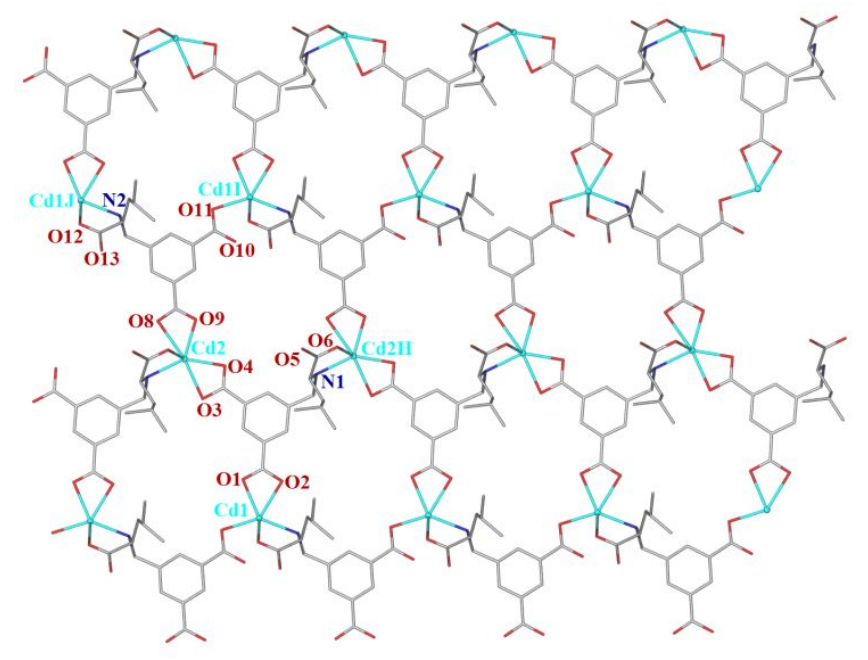

Figure S13. View of $6^{3}$-network formed by the two independent $\left(\mathrm{L}^{2}\right)^{3-}$ ligands linking with $\mathrm{Cd} 1$ and $\mathrm{Cd} 2$ centers in $\mathbf{3}$, with coordination water molecules being omitted for clarity. Symmetry code: $(\mathrm{H}) x,-1+y, z$; (I) $-1+x, y, z$; (J) $-1+x, 1+y$, $z$. 


\section{Left-handed helix}

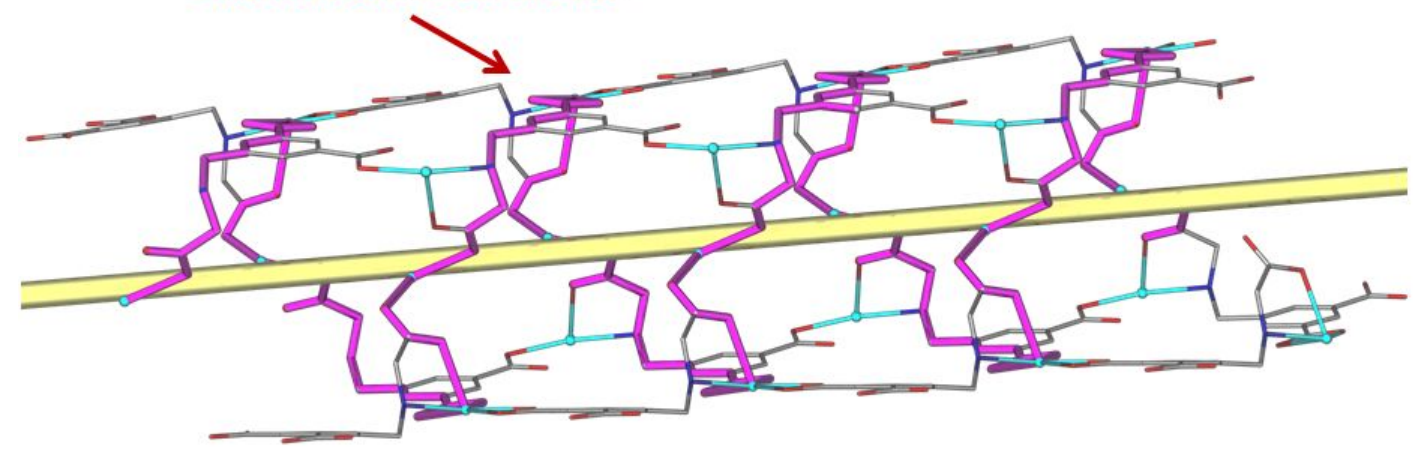

\section{Right-handed helix}

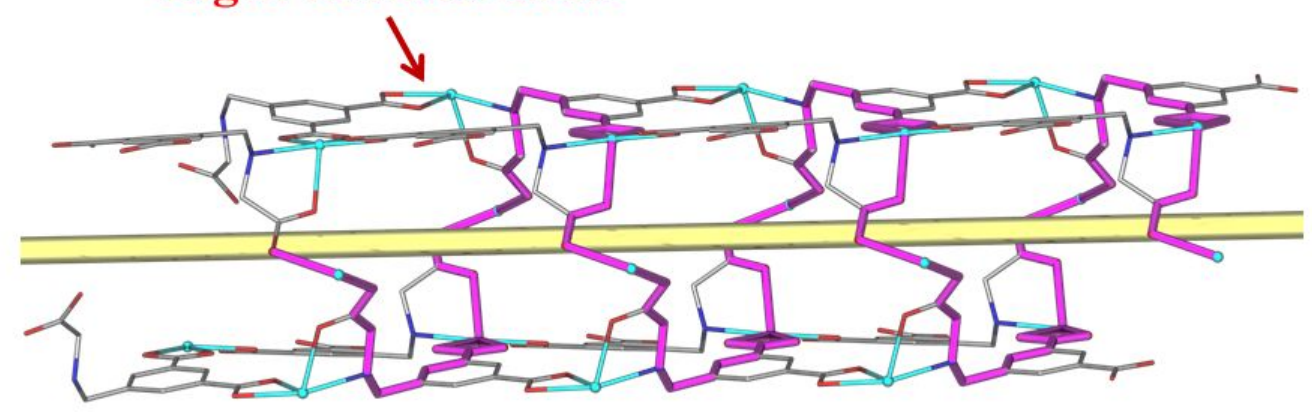

Figure S14. View of $b$-axially extending left- and right-handed $2_{1}$ helixes in the double-layer framework containing symmetry-related $\mathrm{Cd} 1, \mathrm{Cd} 2$, and $\mathrm{Cd} 3$ in $\mathbf{3}$, with coordination water molecules and the isobutyls of amino acid groups in ligands $\left(\mathrm{L}^{2}\right)^{3-}$ being omitted for clarity.

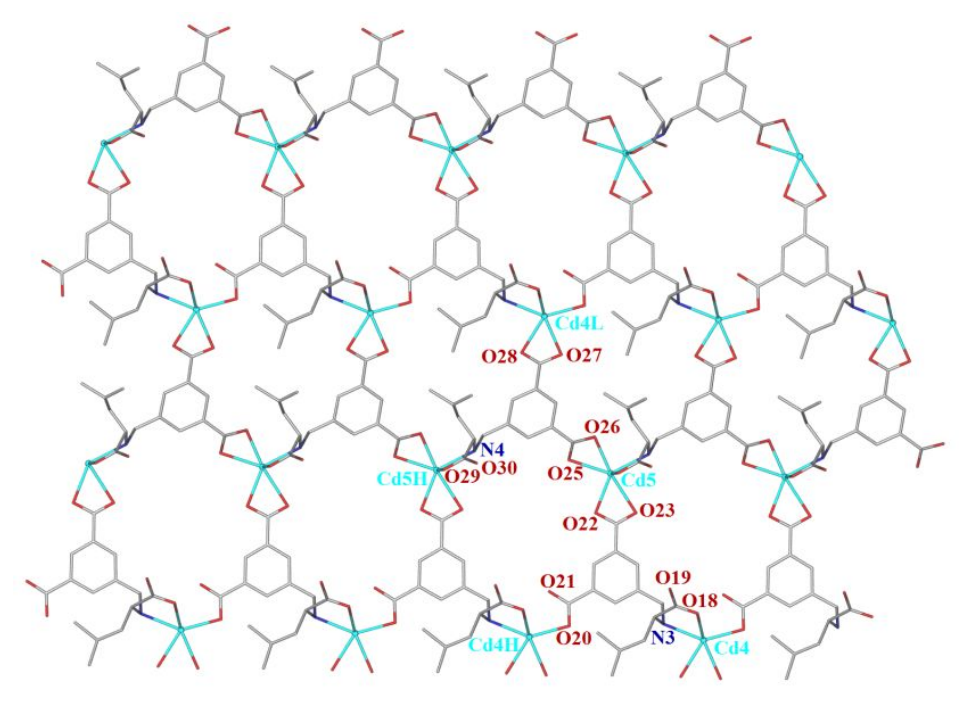

Figure S15. View of $6^{3}$-network formed by the two independent $\left(L^{2}\right)^{3-}$ ligands linking with $\mathrm{Cd} 4$ and $\mathrm{Cd} 5$ centers in $\mathbf{3}$, with coordination water molecules being omitted for clarity. Symmetry code: (H) $x,-1+y, z$; (L) $-1+x,-1+y, z$. 


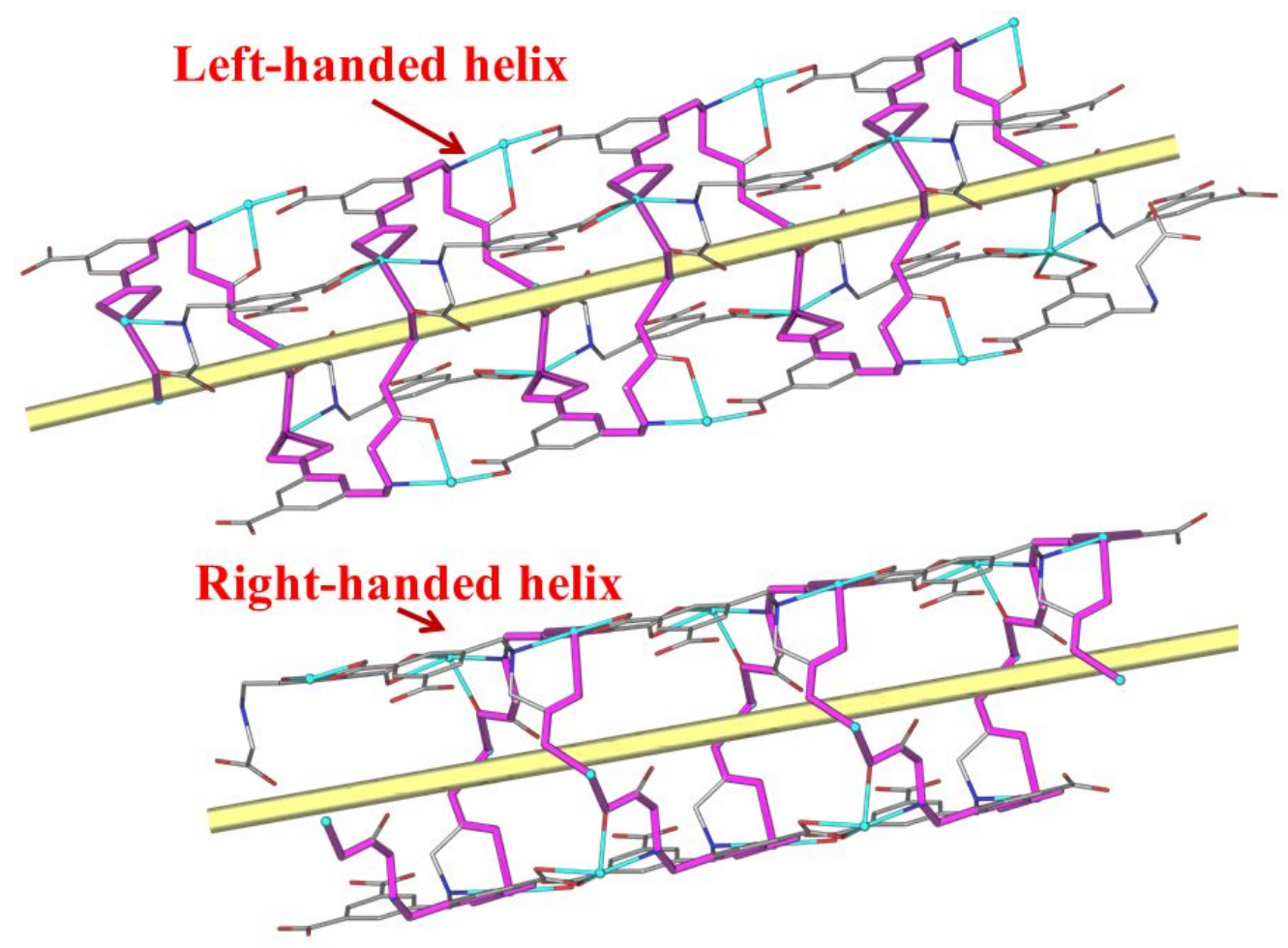

Figure S16. View of $b$-axially extending left- and right-handed $2_{1}$ helixes in the double-layer framework containing symmetry-related $\mathrm{Cd} 4, \mathrm{Cd} 5$, and $\mathrm{Cd} 6$ in $\mathbf{3}$, with coordination water molecules and the isobutyls of amino acid groups in ligands $\left(\mathrm{L}^{2}\right)^{3-}$ being omitted for clarity.

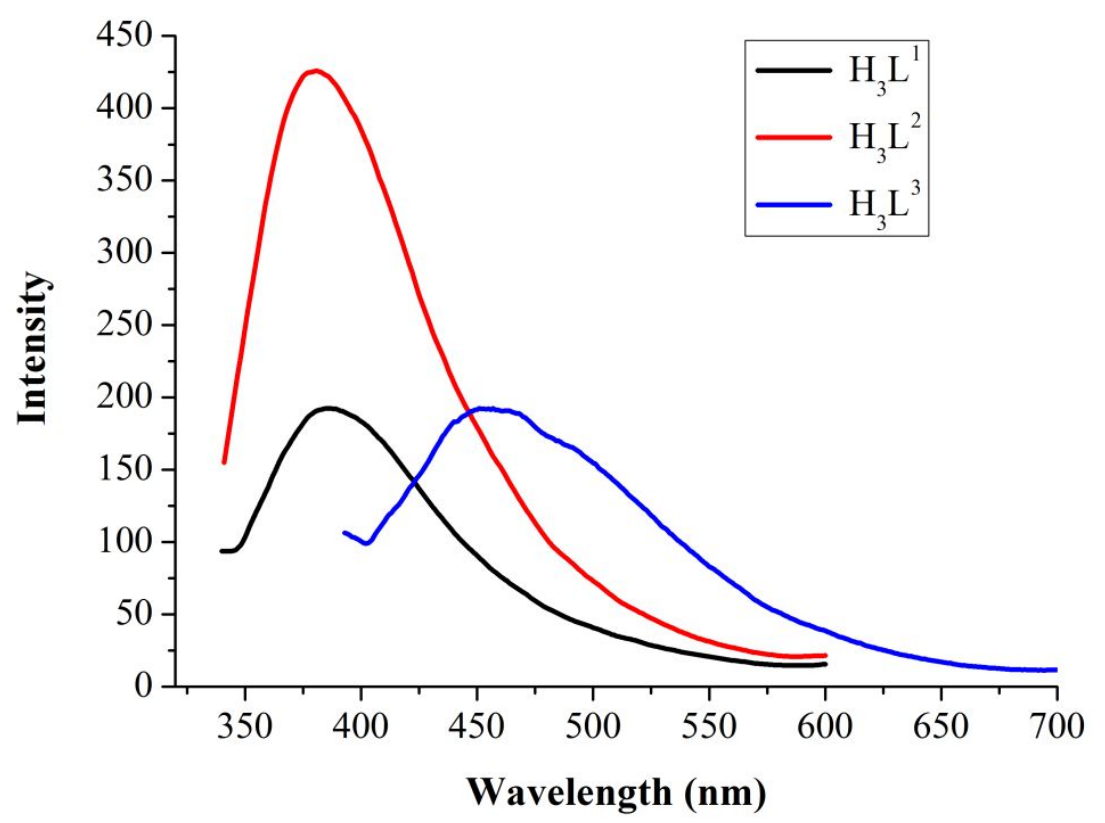

Figure S17. Solid-state fluorescent behaviors of free ligands $\mathrm{H}_{3} \mathrm{~L}^{1}-\mathrm{H}_{3} \mathrm{~L}^{3}$ at room temperature excited at 310, 309 and $373 \mathrm{~nm}$, respectively. 


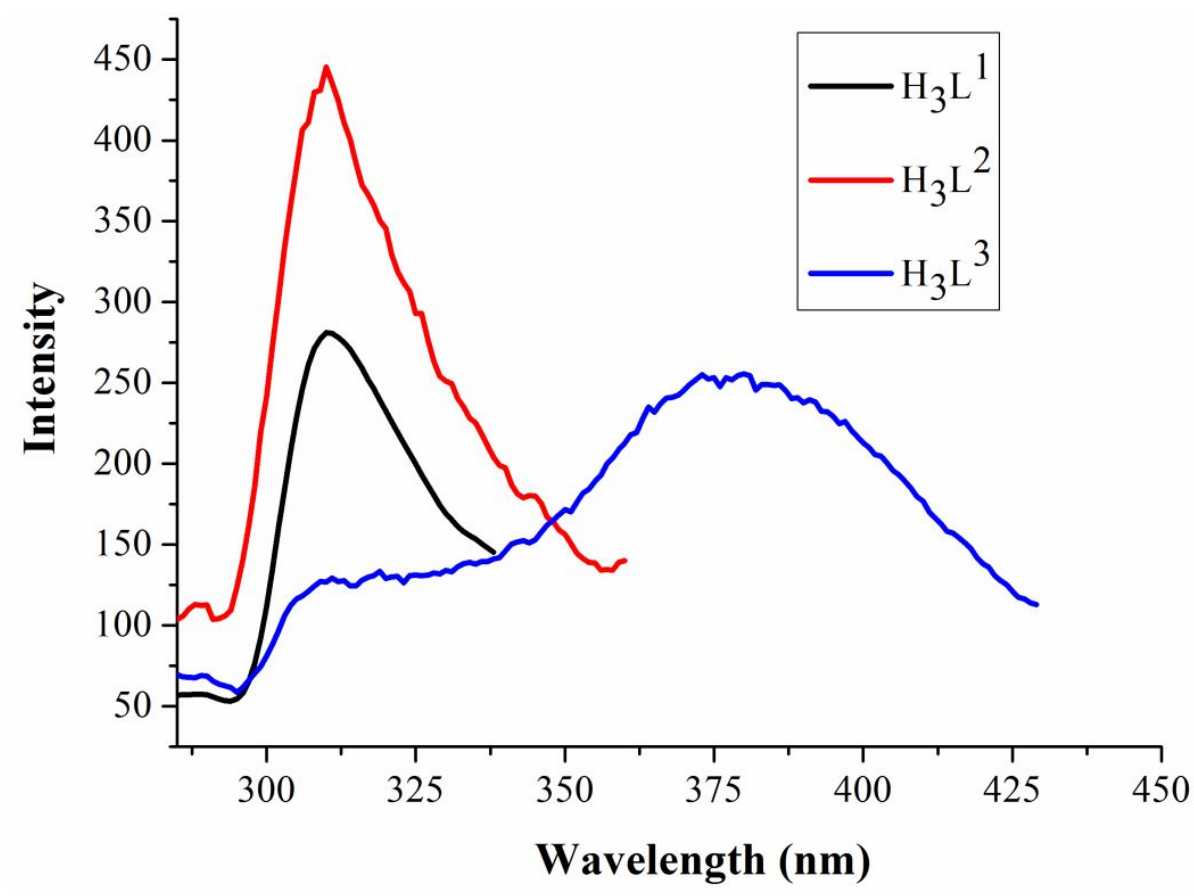

Figure S18. Solid-state excitation spectra of free ligands $\mathrm{H}_{3} \mathrm{~L}^{1}-\mathrm{H}_{3} \mathrm{~L}^{3}$ at room temperature.

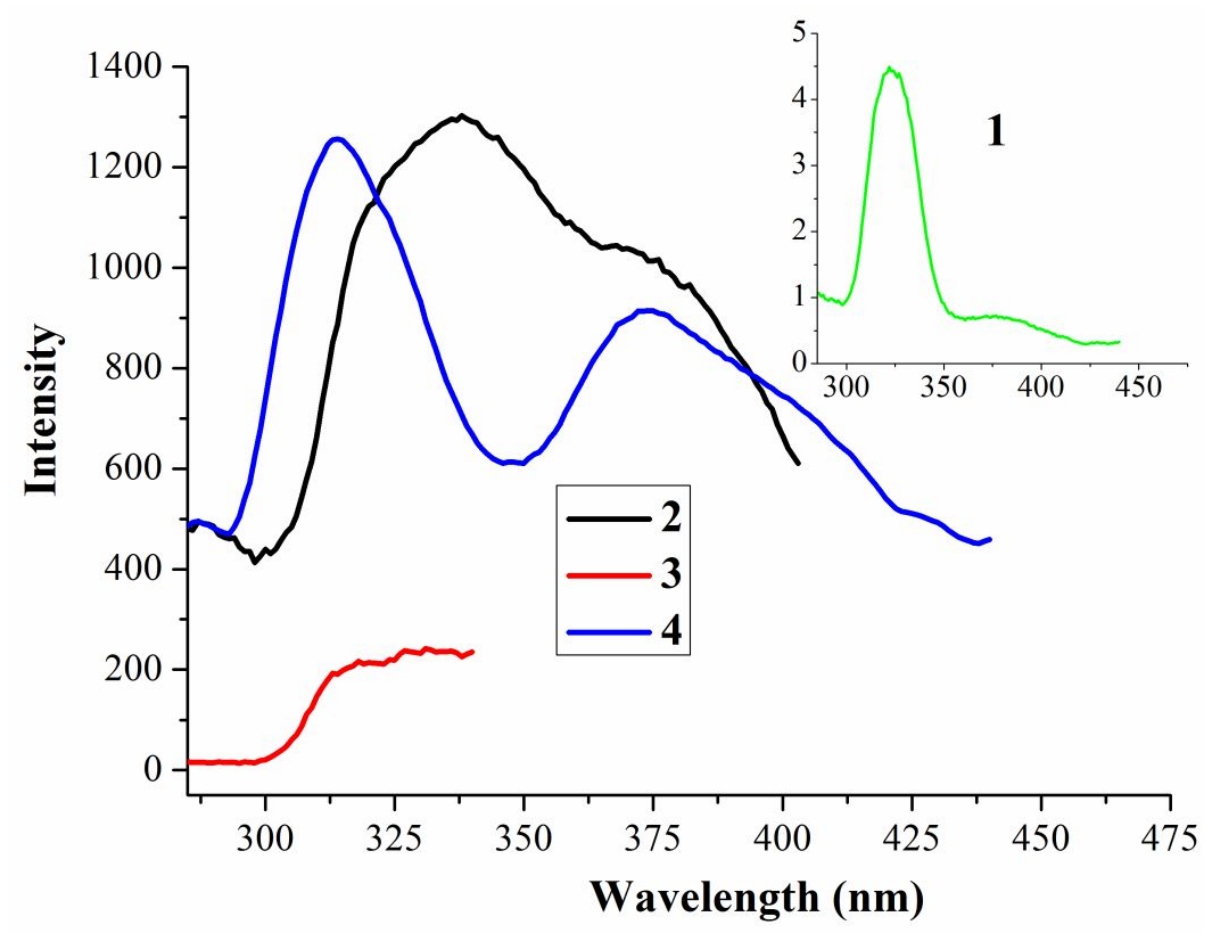

Figure S19. Solid-state excitation spectra of free ligands 1-4 at room temperature. 


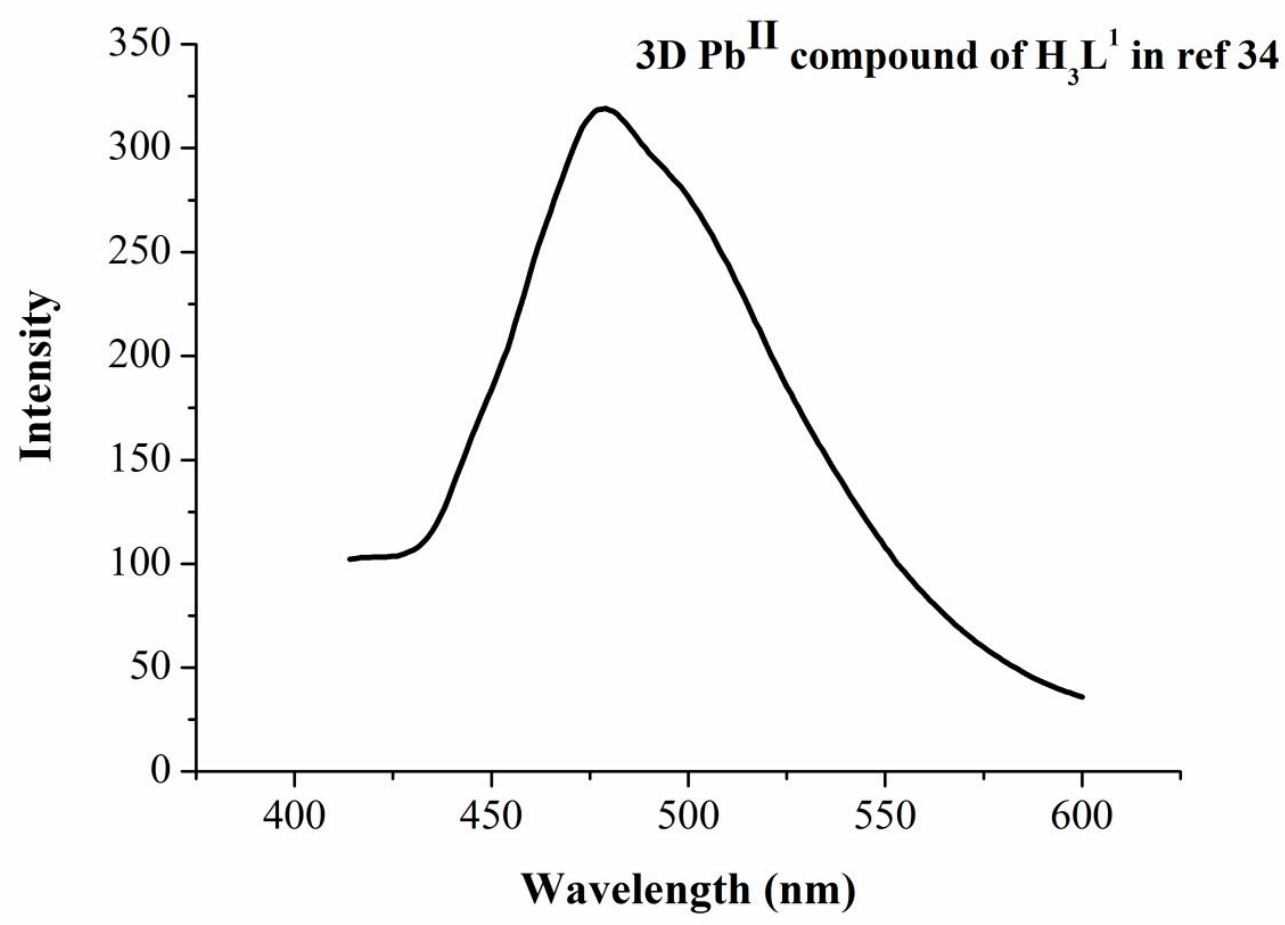

Figure S20. Solid-state fluorescent behavior of $3 \mathrm{D} \mathrm{Pb}$ (II compound of $\mathrm{H}_{3} \mathrm{~L}^{1}$ in ref 37 at room temperature.

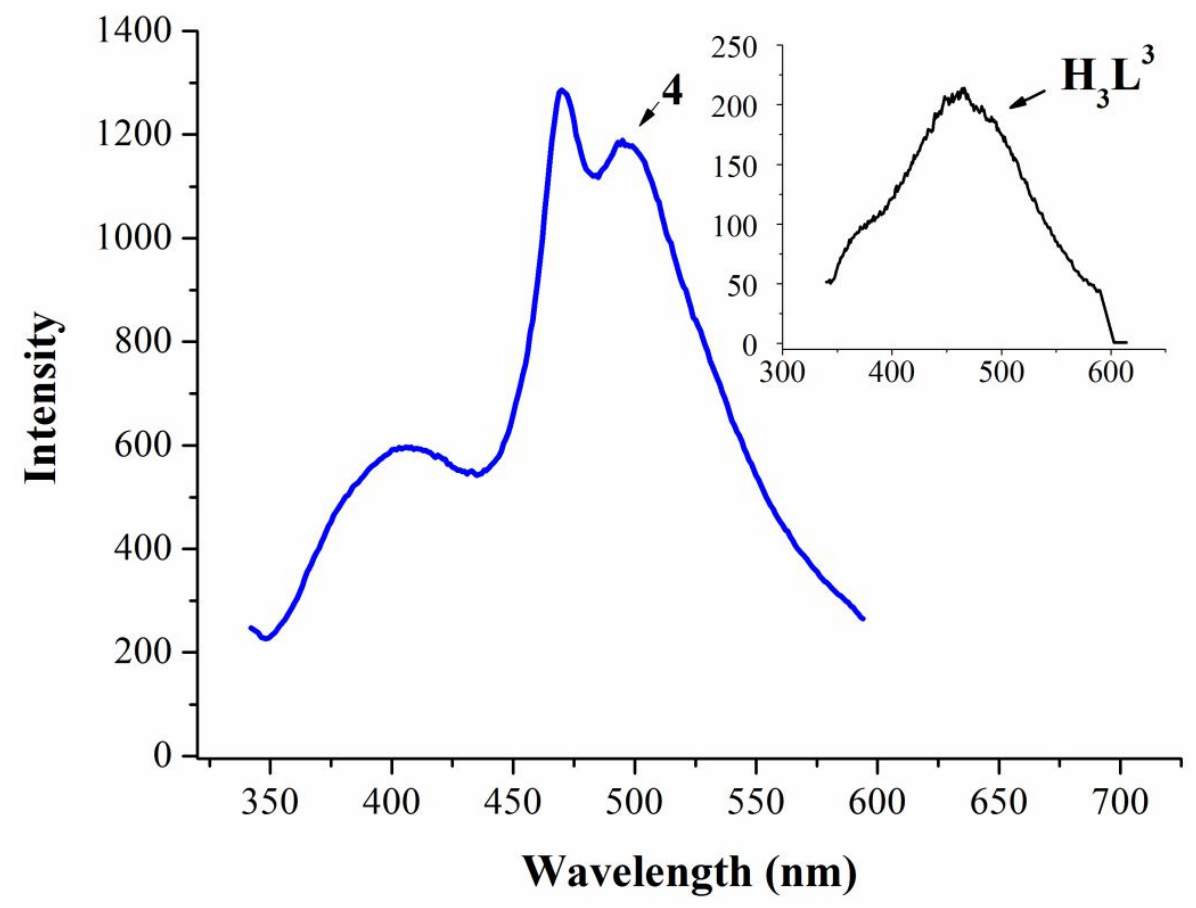

Figure S21. Solid-state fluorescent behaviors of free ligands $\mathrm{H}_{3} \mathrm{~L}^{3}$ and its complex 4 at room temperature excited at 310 and $314 \mathrm{~nm}$, respectively. 


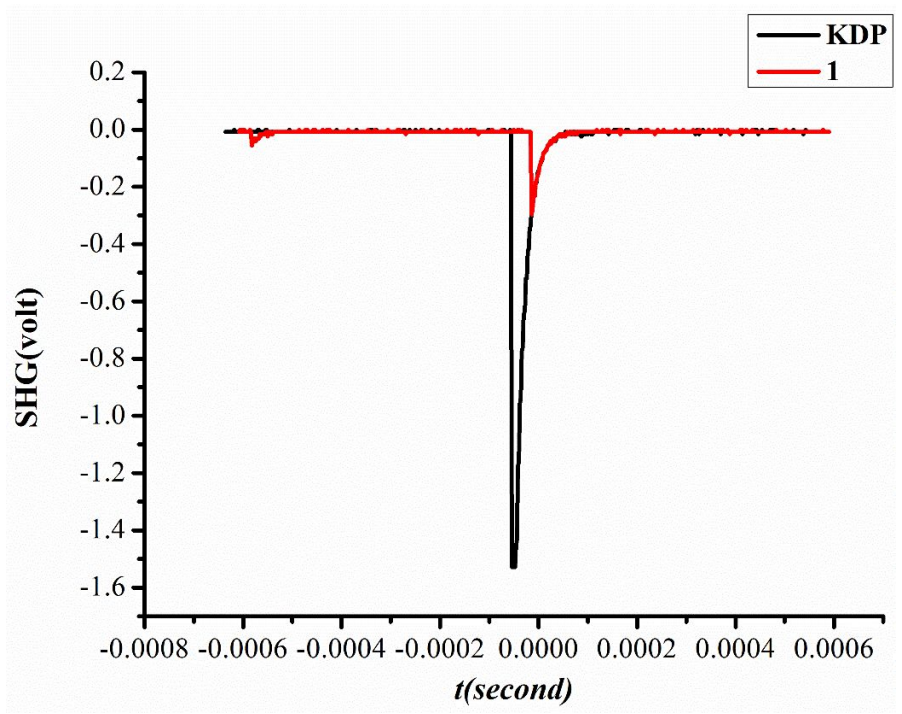

Figure S22. Second-harmonic generation efficiency of $\mathbf{1}$.

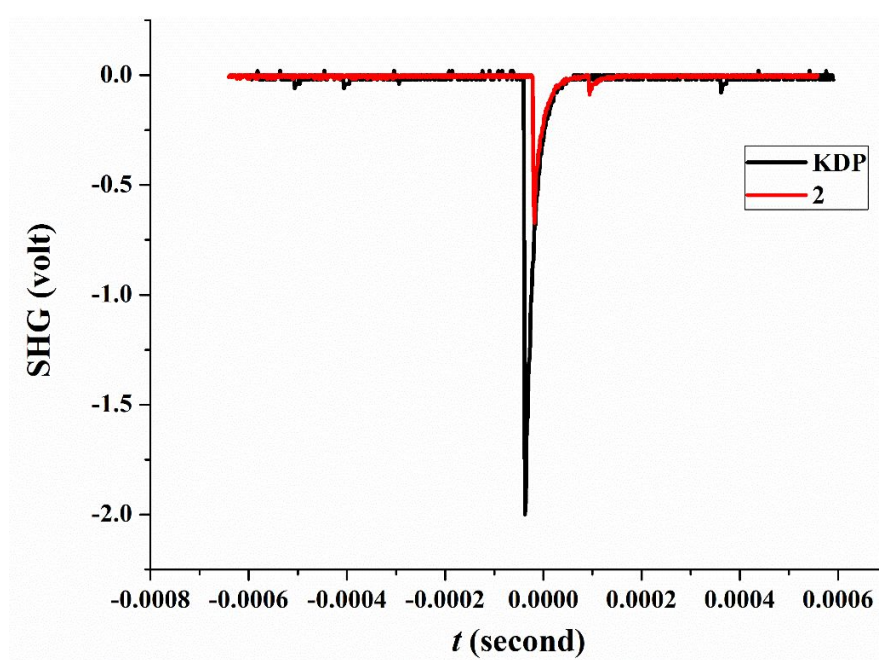

Figure S23. Second-harmonic generation efficiency of 2.

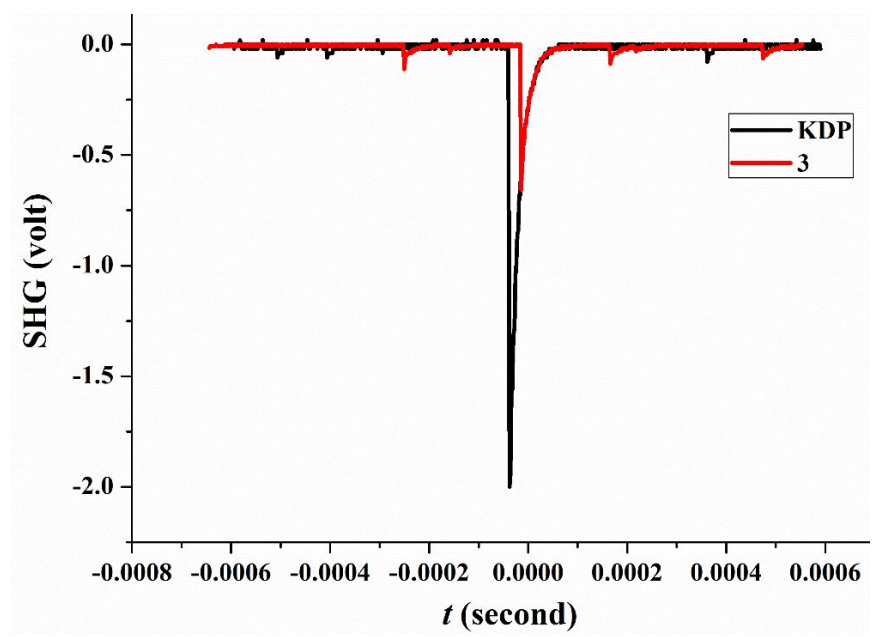

Figure S24. Second-harmonic generation efficiency of 3. 